NISTIR 7724

\title{
A Next Generation Electrodynamic Shaker for the Primary Calibration of Accelerometers
}

\section{The NIST and AFMETCAL Super Shaker Project}

Bev Payne

Kari K. Harper 



\section{A Next Generation Electrodynamic Shaker for the Primary Calibration of Accelerometers The NIST and AFMETCAL Super Shaker Project}

Bev Payne

Kari K. Harper Manufacturing Engineering Laboratory Manufacturing Metrology Division 

Table of Contents

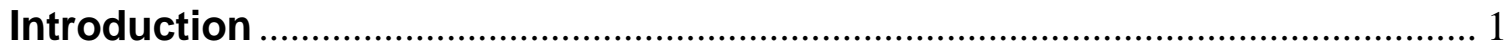

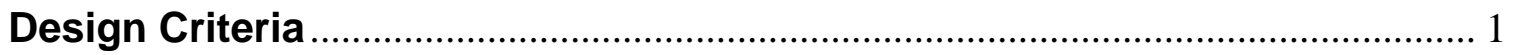

Description of the Shaker Assembly ................................................................. 2

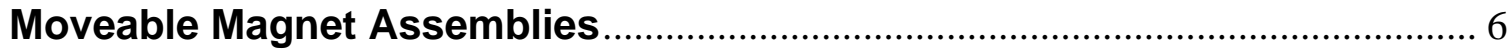

Moving Element and Carriage Description ........................................................ 7

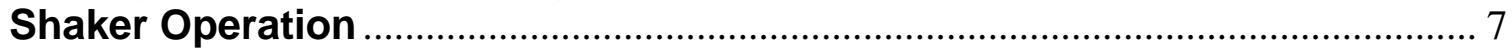

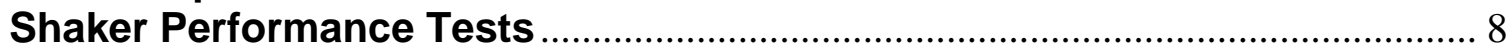

Calibration by Reciprocity ............................................................................... 10

Calibration by Laser Fringe-Counting Interferometry....................................... 12

Calibration by Minimum-Point Method Laser Interferometry .......................... 13

Calibration by Sine-Approximation Method Using Quadrature Laser

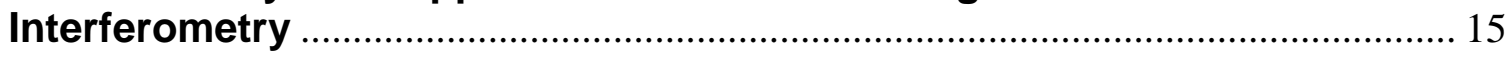

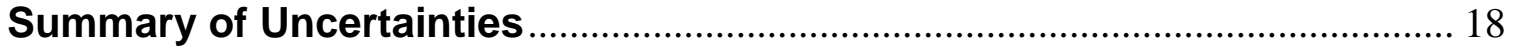

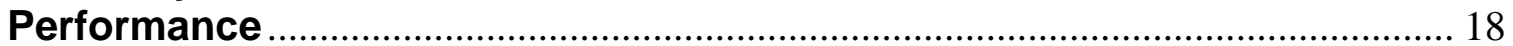

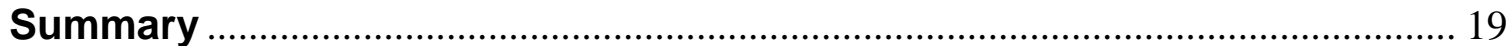

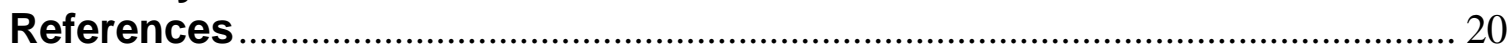

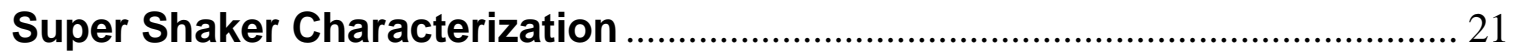





\title{
A Next Generation Electrodynamic Shaker for the Primary Calibration of Accelerometers
}

\author{
The NIST and AFMETCAL Super Shaker Project \\ Bev Payne, Guest Researcher, NIST \\ Kari K. Harper, Mechanical Engineer, NIST
}

\begin{abstract}
Introduction
A new shaker system was designed to allow direct primary calibration of accelerometers using both reciprocity and interferometer techniques over the frequency range $40 \mathrm{~Hz}$ to $10,000 \mathrm{~Hz}$. The system uses dual magnets and a dualcoil moving element. The design uses damped flexures to support the moving element. The dual-coil feature eliminates the need for an additional shaker for reciprocity calibrations. To accommodate improved comparison calibrations, the moving element is compact in design and is equipped with axially-oriented mounting tables at each end. Provision of easy laser interferometer beam access to both ends of the moving element allows interferometric accelerometer calibrations.

In order to maximize the potential for design optimization, two Super Shaker systems were built: Super Shaker I located at the Air Force Metrology Laboratory (AFMETCAL) Heath, OH, and Super Shaker II located at NIST. The design also allows comparison calibrations to be performed over this same frequency range. This report gives performance data for both shakers, with the AFMETCAL shaker referred to in this report as SS1, and the NIST shaker as SS2. The SS2 shaker was the second shaker constructed and incorporates minor changes from the original design. The principal change was a more flexible flexure system for the moving element support.
\end{abstract}

\section{Design Criteria}

Primary calibration methods for accelerometers (reciprocity and laser interferometry) require high purity of the displacement signal wave form. This is especially true for reciprocity calibrations. For reciprocity measurements, the procedure does not take into account the fact that the accelerometer is measuring motion in only one direction, but rather the calculations involve measurement of current differences in the drive coil. Therefore, even a small amounts of distortion and cross-axis motion adversely affect the reciprocity calibration. A general rule is that cross-axis motion should be less than $5 \%$ and harmonic distortion less than $2 \%$ in order to perform reciprocity calibrations at 1 to $2 \%$ uncertainty. For laser interferometer fringe-counting calibration, only the 
on-axis displacement amplitude is measured. Provided that the laser beam/interferometer is properly aligned, the cross-axis motion of the shaker is not as significant in a fringe-counting calibration as in the reciprocity calibration. In designing the Super Shaker $[1,2]$, care was taken to minimize both harmonic distortion and cross-axis motion. Minimization of the latter was especially important to our goal of improving reciprocity calibrations. Among the critical factors considered were:

1. Magnet dimensions, especially the air gap size, roundness, symmetry, and finish.

2. Moving element dimensions, including diameter, symmetry, and roundness.

3. Position and repeatability of position of the moving element in the magnetic gap assembly.

4. Symmetry of coil windings and uniformity of coil wire.

5. Suspension system for the moving element in the magnetic gap assembly.

6. Isolation of critical parts of the shaker to prevent cross coupling of various vibrating parts of the assembly.

The dimensional tolerances for all Super Shaker assemblies were specified to be no greater than $30 \mu \mathrm{m}$ (0.001 inch).

\section{Description of the Shaker Assembly}

The Super Shaker, shown in Fig. 1, is $0.64 \mathrm{~m}$ (25 in) wide, $1.22 \mathrm{~m}$ (48 in) long, by about $0.5 \mathrm{~m}$ (20 in) high. It is mounted on soft rubber pads on a heavy flat table, pneumatically isolated from ground vibration.

The frame consists of three supporting pedestals, one at each end and one in the middle, and four large diameter shafts running end to end, two clamped near the bottom of the three pedestals, and two clamped near the top of the pedestals. Two magnet assemblies, equipped with air bearings, are supported by the two horizontal rods which are the upper shafts of the frame assembly. The air bearings allow each magnet assembly to be moved away from the moving element to provide the access needed for attachment of accelerometers or masses. The operating axis is parallel to the upper shafts and centered in the plane bisecting them.

A common cause of calibration inaccuracy is interference from frame resonances. The interferences are resisted by internal dampers inside the three pedestals and by damping inside the four shafts. The pedestals and shafts are 
also filled with sand to further dampen any vibration in the frame. These interferences are further resisted by the large magnet mass, and small resulting motion, as compared to the moving element mass. The small magnet motions are isolated from the frame by rubber pads.

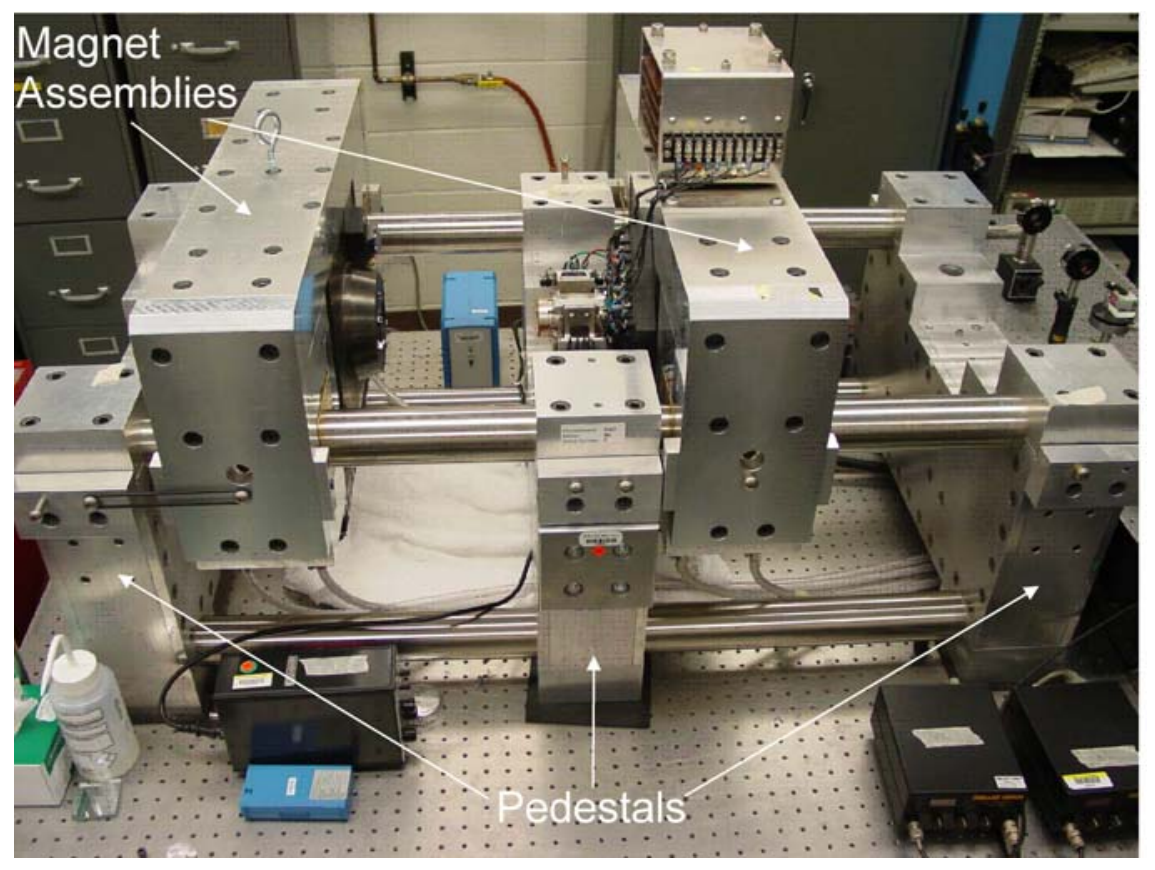

Figure 1a. SS2 at NIST

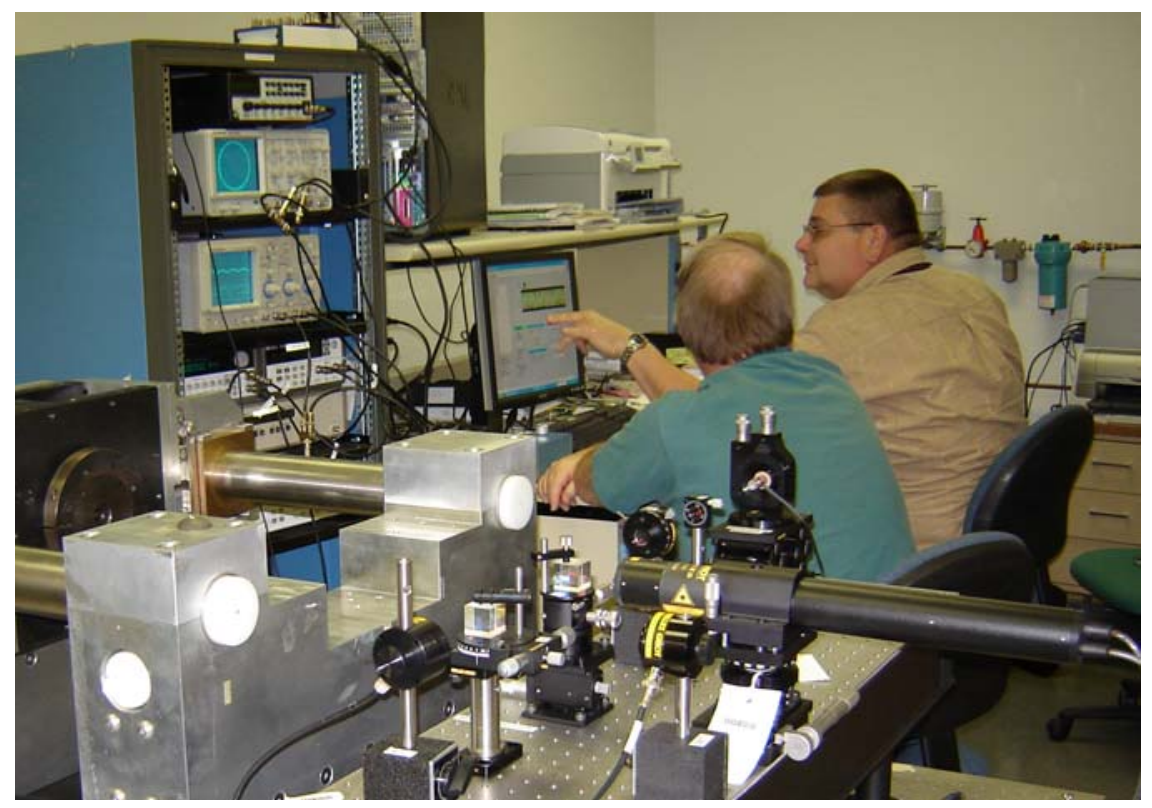

Figure 1b. SS1 with instrumentation and optical components of laser interferometer at AFMETCAL, Heath, $\mathrm{OH}$ 


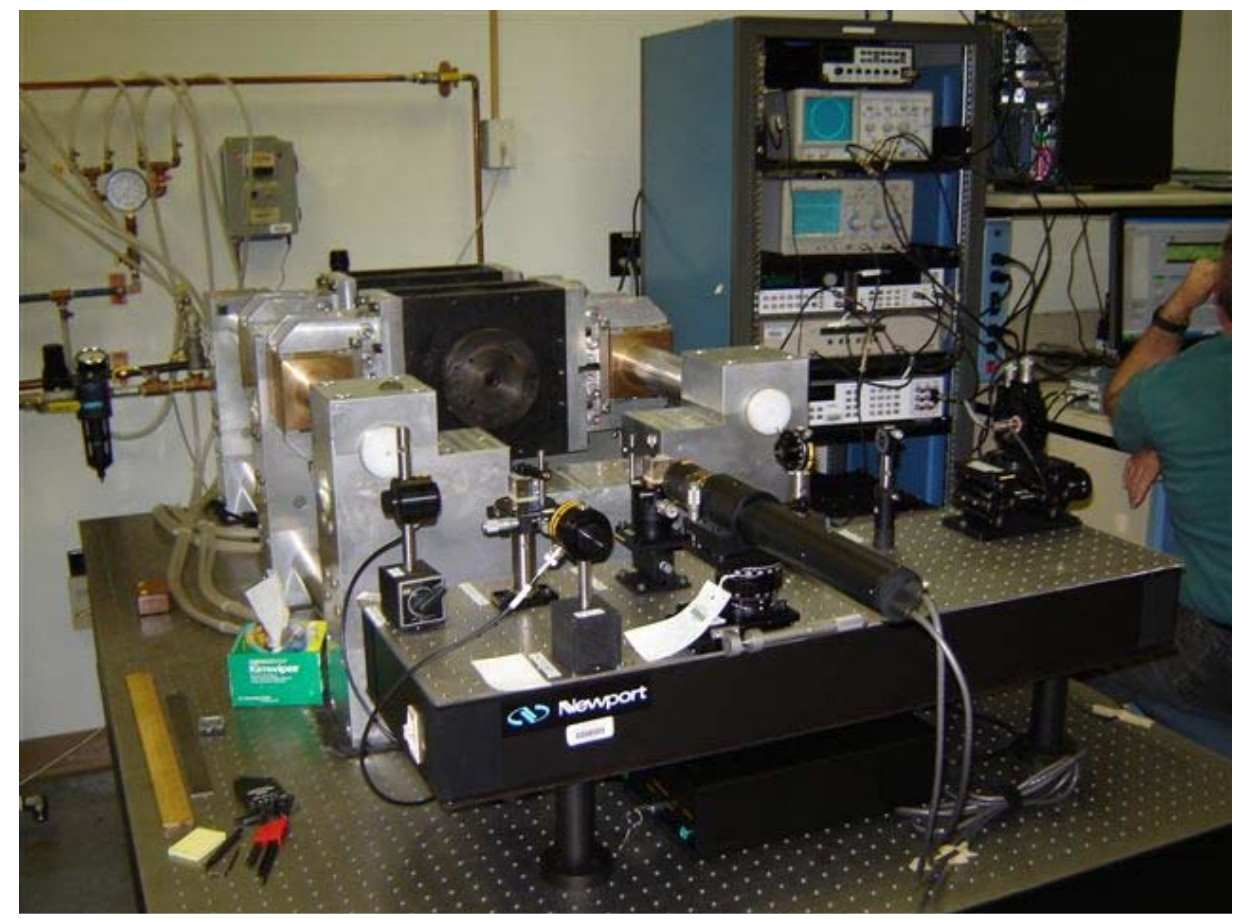

Figure 1c. SS1 mounted on isolation table at AFMETCAL, Heath, OH

A carriage on the middle pedestal supports the moving element, centered on the operating axis. The moving element is symmetrical, with coils on each end. Machined from a solid aluminum block, the moving element, shown in Fig. 2, is symmetrically shaped with respect to a bisecting plane perpendicular to the operating axis. The moving element, supported by damped flexures attached to the pedestal in the middle, carries the accelerometer to be calibrated.

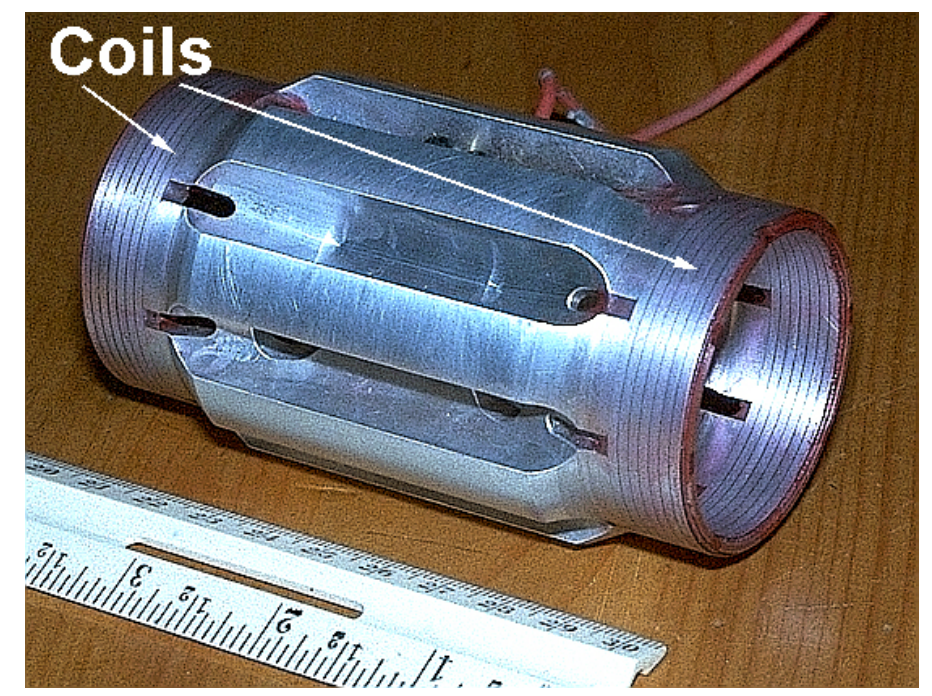

Figure 2. Moving element of SS1 
Placement of the specimen (accelerometer) mounting tables at the ends of deep central cavities of the moving element allows specimens to be mounted close to the bisecting plane and inside the envelopes of the coils located at each end.

The outer structure between the two coils is enlarged to provide axial stiffness for high frequency operation. The shaker is equipped with removable mounting tables (or cones), made from beryllium with a nickel coating to prevent scratching, Fig. 3a. The cones have threaded steel inserts for mounting accelerometers. The beryllium mounting cones extend the frequency range of the shaker to $10,000 \mathrm{~Hz}$. Special wrenches were designed to enable easy removal or attachment of the cones to the moving element, Fig. 3b and 3c.

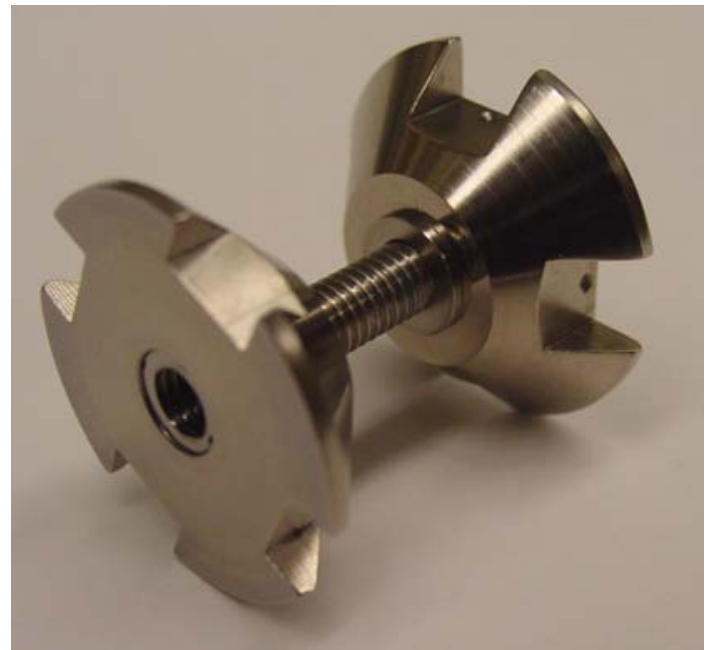

Figure 3a. Nickel-coated beryllium mounting tables for the moving element, shown with titanium threaded inserts

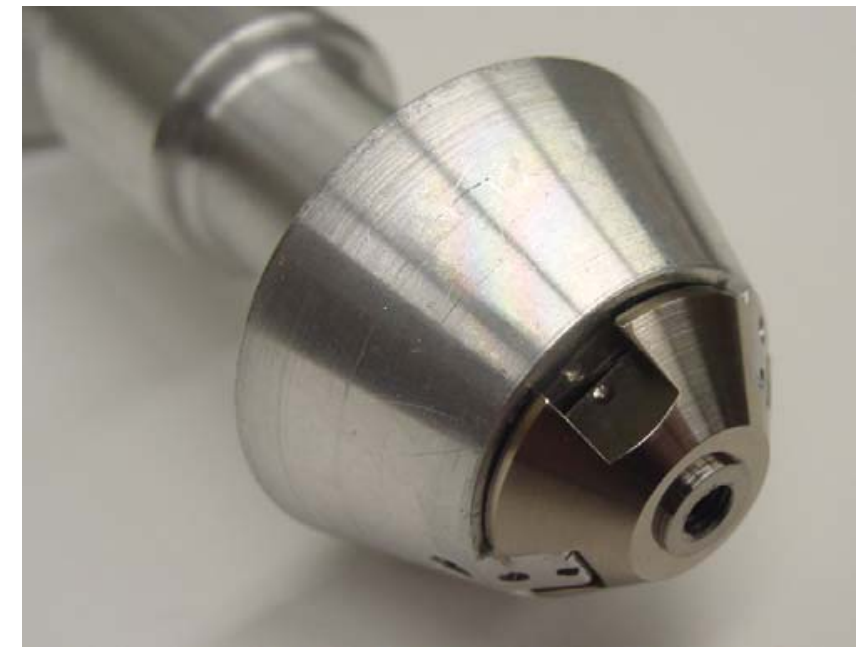

Figure $3 \mathrm{~b}$. Wrench for attaching and removing mounting tables, shown with table on wrench

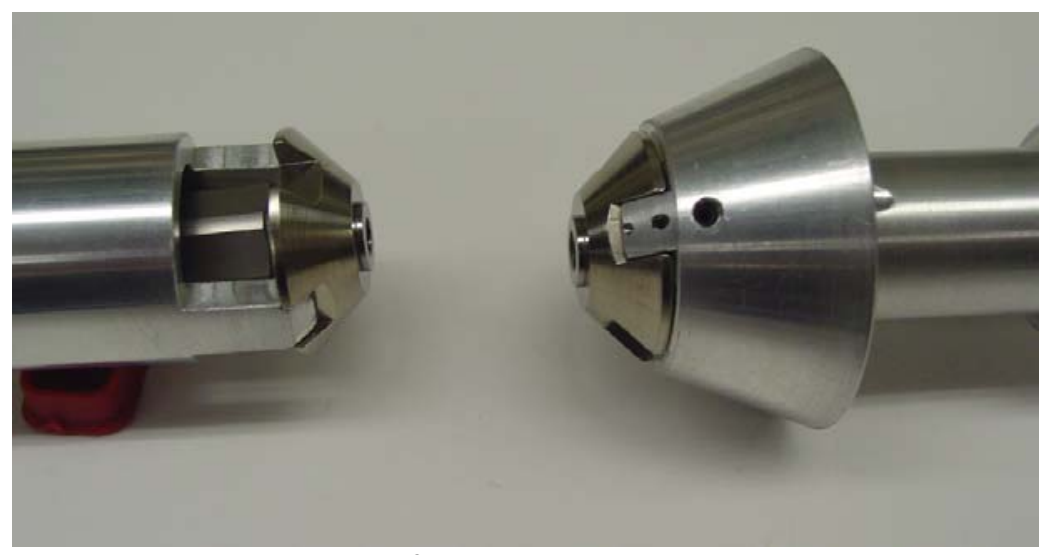

Figure 3c. Wrenches for attaching and removing mounting tables, shown with tables loaded on wrench 


\section{Moveable Magnet Assemblies}

The magnet assembly is shown in Fig. 4. Each magnet assembly has two major parts: the outer permanent magnet and an inner air gap assembly. The mass of the permanent magnets, $68 \mathrm{~kg}$, has been maximized within practical constraints in order to minimize the motion of the magnet assembly relative to the moving element, $225 \mathrm{~g}$. This reduces interference to the rectilinear motion of the moving element. Residual magnet motions are isolated from the frame by rubber pads.
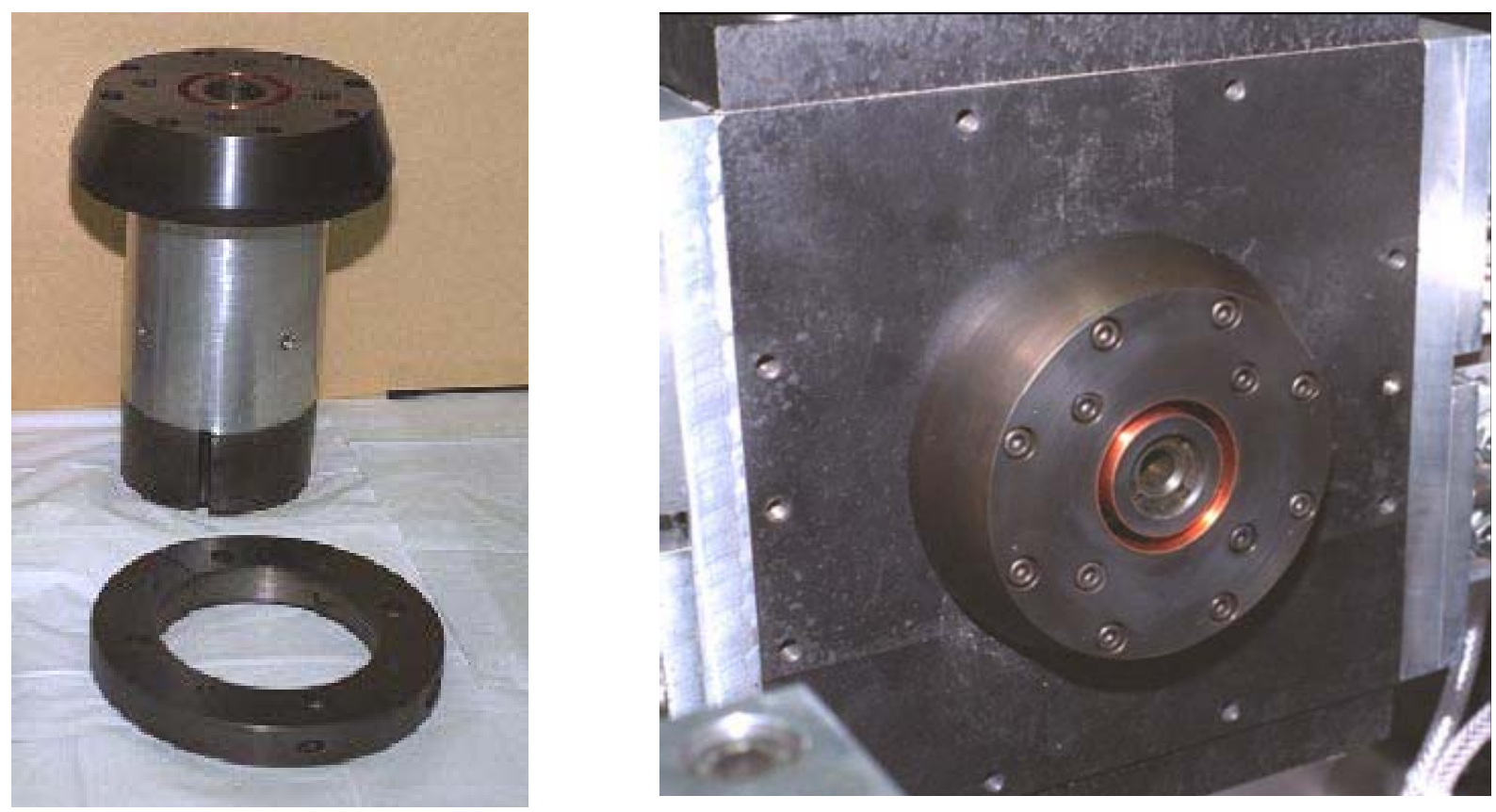

Figure 4. Inner gap assembly (left) and complete magnet assembly (right)

Each air gap assembly is equipped with inner and outer copper shading sleeves, a large axial port for the accelerometer cable, and four small ports for interferometer beam access. Each assembly is also equipped with additional ports for air cooling as needed. The air gap assemblies are de-mountable to facilitate installation of new assemblies of different air gap sizes, should future applications require moving elements of different size. Magnetic keepers are provided to allow this to be done without demagnetizing the permanent magnets. 


\section{Moving Element and Carriage Description}

The moving element and carriage assembly are shown in Fig. 5, attached to the middle pedestal of the shaker. The length of the assembly is $101.6 \mathrm{~mm}(4 \mathrm{in})$ and the diameter is $63.5 \mathrm{~mm}(2.5 \mathrm{in})$. The structure is aluminum and the mounting tables are of beryllium as described above, and are $25.4 \mathrm{~mm}$ (one inch) in diameter. The first axial resonance is approximately $22 \mathrm{kHz}$. The diameter of the mounting table is adequate to allow the laser beam the access required for multiple-reflection interferometer calibrations.

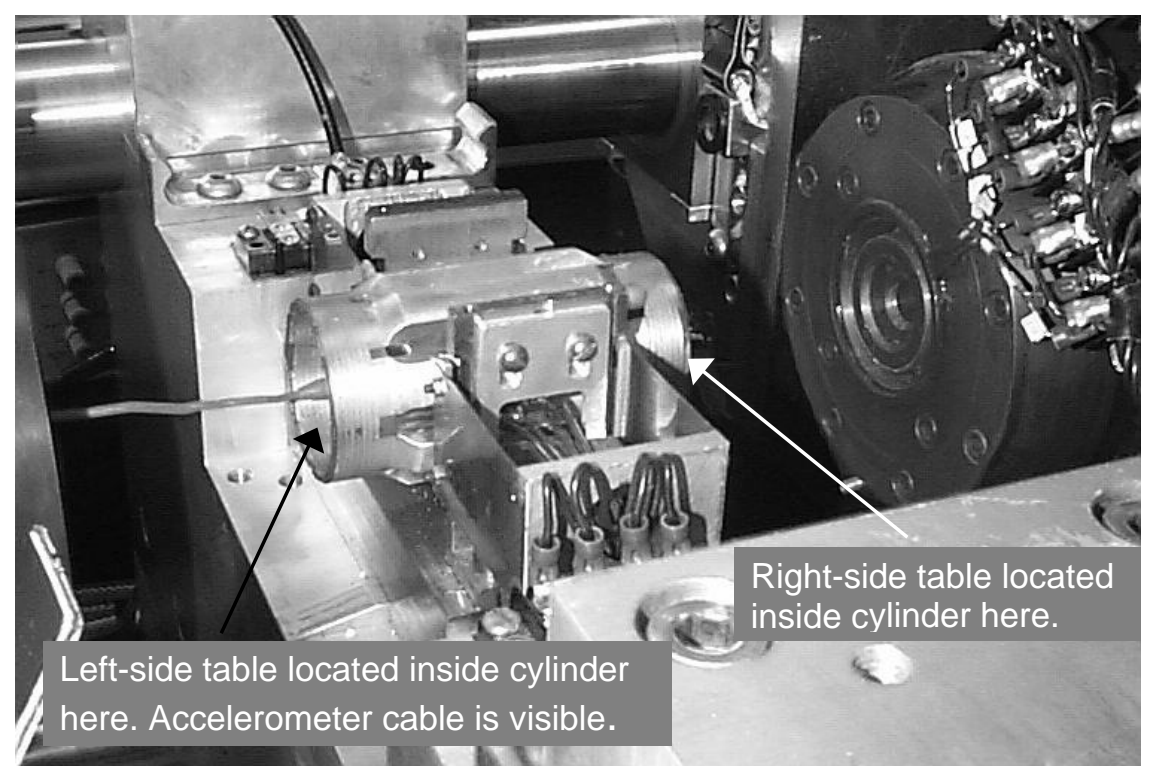

Figure 5. Moving element and carriage assembly, shown with accelerometer attached

To maximize versatility, the four laser beam ports provided in each air gap assembly for this purpose are positioned at 90 degree intervals.

The carriage of the Super Shaker supports the moving element on four damped beryllium copper flexures. These flexures establish the rest position of the moving element, guide its motion along the system axis, and resist motions perpendicular to the system axis.

\section{Shaker Operation}

The vibratory drive force for the moving element is generated by an alternating current in one or both of the drive coils in the air gap magnetic fields. For reciprocity calibrations, only one moving element coil is driven. For interferometric or comparison calibrations, either or both of the coils may be driven. Both coils are driven when large displacements and accelerations are desired. 
A low-distortion power amplifier amplifies a signal from an external signal source to provide the alternating current. As necessary, power amplifier heating is reduced by connecting high-power resistors in series with the amplifier output. The use of these resistors is important for high frequency interferometric operation and critical for pulse operation. The current applied to the drive coil(s) must have low levels of harmonic distortion in order to minimize interference caused by excitation of high frequency (typically above $20 \mathrm{kHz}$ ) resonances of the accelerometer or moving element.

\section{Shaker Performance Tests}

Extensive performance tests were conducted on shakers SS1 and SS2. These tests examined harmonic distortion, cross-axis motion, relative motion between the two mounting surfaces of the moving element. unwanted movement of the optics relative to the shaker, and random effects on repeated measurements (repeatability). The first three of these factors were observed to have a significant influence on the uncertainty of calibrations performed on this system. Tests and results for these factors are detailed below.

A Fast Fourier Transform (FFT) signal analyzer was used to measure the harmonic distortion components of an accelerometer mounted on the shaker. The tests were run at approximately $20 \mathrm{~m} / \mathrm{s}^{2}\left(2 g_{\mathrm{n}}{ }^{1}\right)$ and the maximum harmonic component at each frequency is shown in Fig. 6. Harmonic distortion can be minimized by careful design of the moving element and magnet structure, as described in the Design Criteria section above.

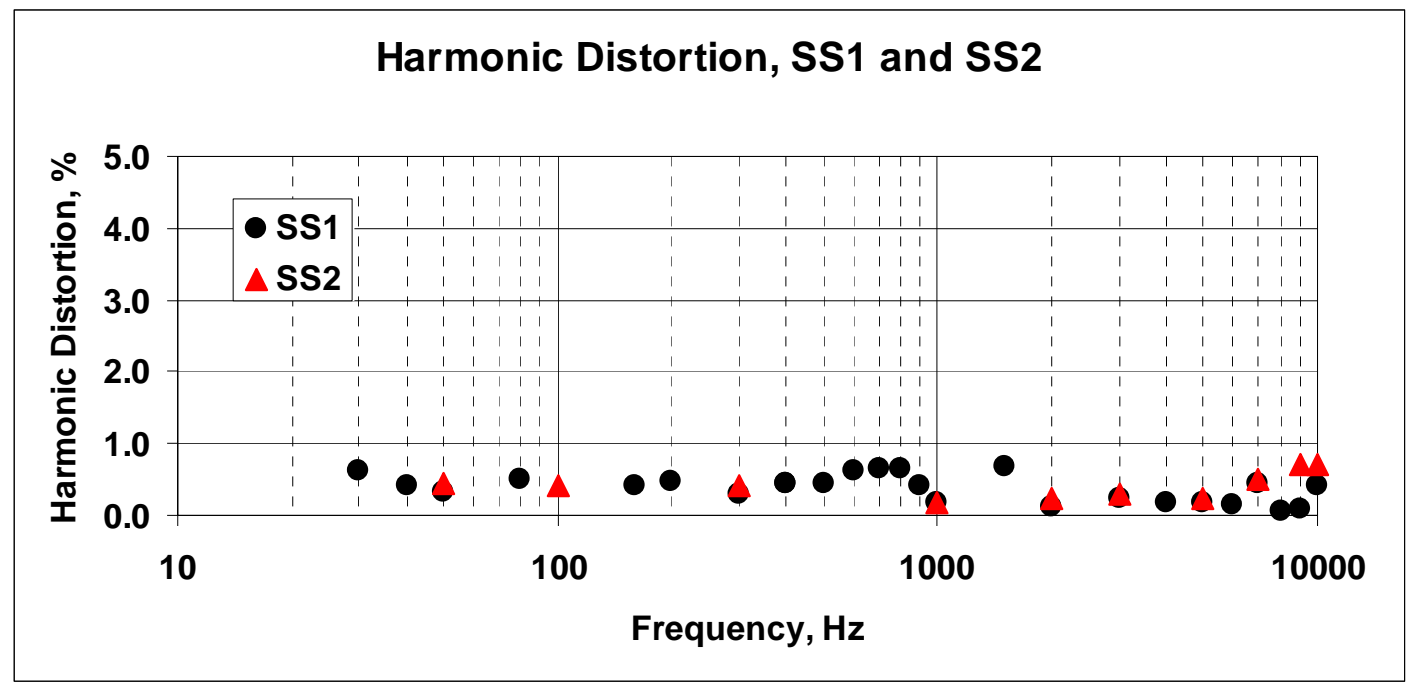

Figure 6. Total harmonic distortion for SS1 and SS2

${ }^{1} g_{\mathrm{n}}=9.80665 \mathrm{~m} / \mathrm{s}^{2}$ 
The cross-axis motion was measured with a small tri-axial accelerometer having a mass of 5 grams. The cross-axis data is plotted in Fig. 7. These data should be considered as an approximate indication of the true cross-axis motion since calibration masses could not be attached to the table at the same time the lightweight accelerometer was attached.

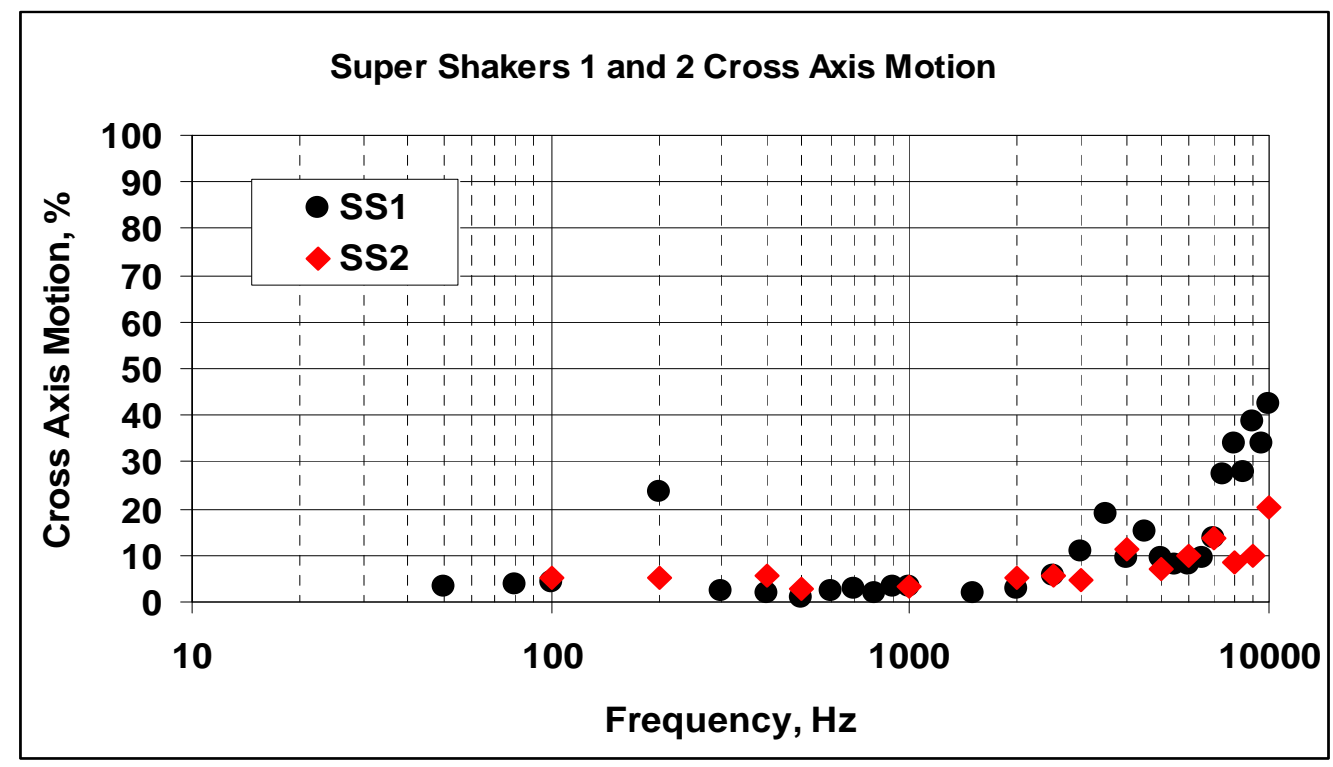

Figure 7. Cross axis motion for SS1 and SS2

Relative motion between the two mounting tables was measured by placing a polished 20 gram tungsten dummy mass on the mounting table where the test accelerometer is normally mounted. A single point laser vibrometer was used to measure the displacement at three positions on the mass, approximately 120 degrees apart. The average displacement taken at the three positions was then compared to the displacement as measured by the quadrature laser interferometer on the other mounting table which has a small mirror attached in the axial center. The differences (\%) in the measured displacement of the two mounting tables are shown graphically in Fig. 8. 


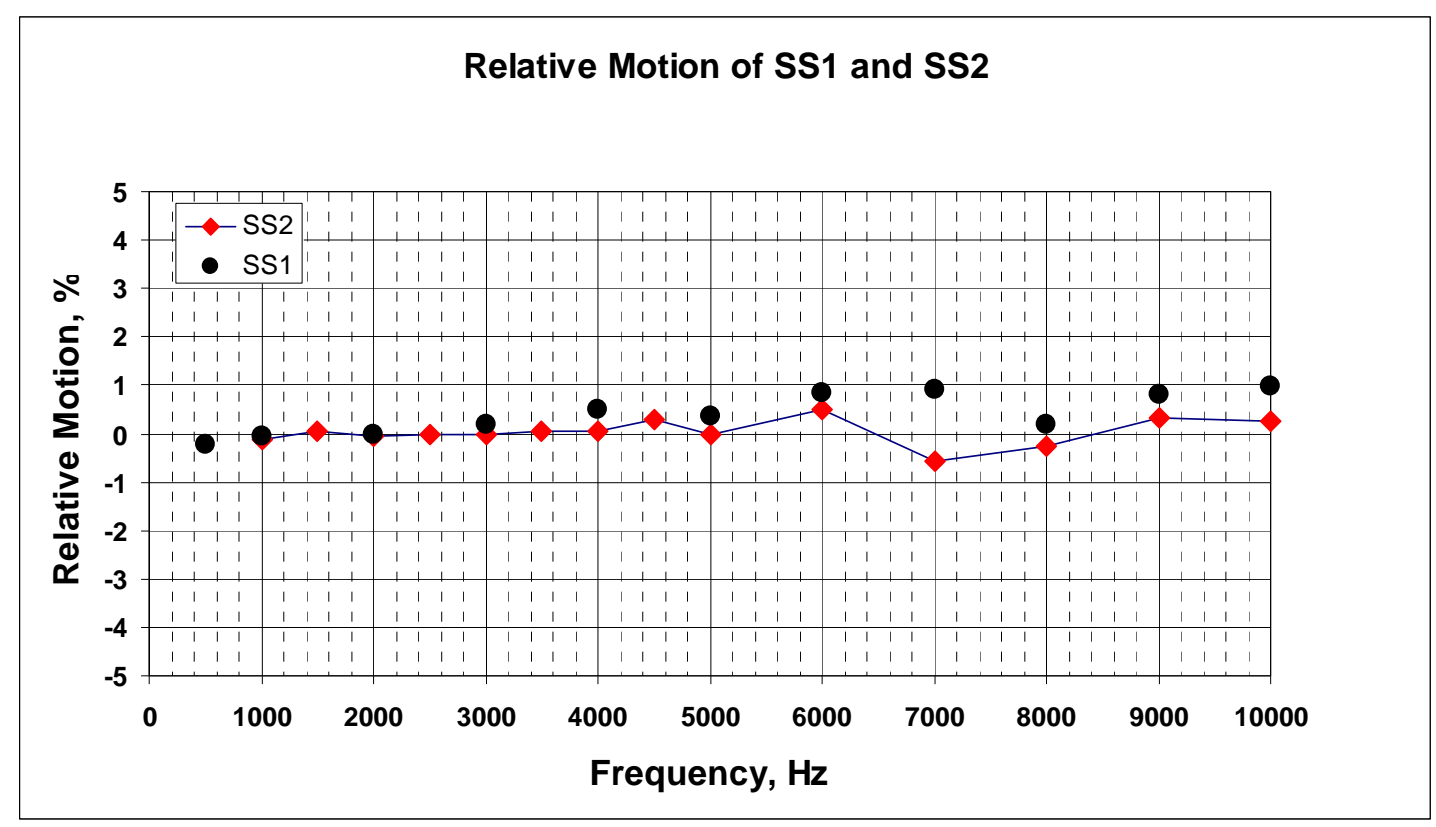

Figure 8. Relative motion between the two mounting tables of the shaker

\section{Calibration by Reciprocity}

Reciprocity calibration of accelerometers has been in use at NIST for many years as a primary method for calibration. It is distinct in that it does not rely on optical instrumentation. It is not based on the wavelength of light, but upon mass for its primary reference. For reciprocity calibrations, a series of five small tungsten carbide precision masses are attached one at a time to the right mounting table, with the accelerometer to be calibrated on the left mounting table (see figure 5). The calibration is briefly describe below, and in more detail in the references listed at the end of this paper [3], [4], and [5].

Since the current in the driver coil is one of the measured quantities, it is necessary to keep the mean position of the moving element constant relative to the magnet. The Super Shaker's moving element operates in a horizontal orientation (figure 5). This avoids the problem encountered in vertical oriented shakers of repositioning the driver coil to the exact same position in the magnetic field when different masses are attached. The flexures of the Super Shaker tend to keep the moving element in approximately the same position relative to the magnet, regardless of the value of the mass attached. Better repositioning of the moving element between mass changes is obtained by delaying the next measurement by a few minutes, to allow the flexures to reposition.

For reciprocity calibration, the test accelerometer is mounted on the left, as shown in Fig. 5; the mirror is removed from the right, and the right mounting table 
is used for attaching the calibration masses. At sufficiently low frequencies the magnitude of the accelerometer sensitivity $(S)$ may be approximated [3-5] by:

$$
S=[(J R) /(2 \pi f)]^{1 / 2},
$$

where:

parameter $J$ equals the zero intercept of a linear fit of $\mathrm{m} /\left(Y_{\mathrm{m}}-Y_{0}\right)$ vs. $\mathrm{m}$, where $Y_{\mathrm{m}}$ and $Y_{0}$ are the measured transfer admittance with and without the calibration mass, $\mathrm{m}$.

The transfer admittance is calculated by dividing the current of the driver coil by the output voltage of the accelerometer while the shaker is energized by the left coil. In a separate measurement, $R$ is the voltage ratio of the output voltage of the accelerometer to the output voltage across the left coil when used as a velocity sensor while the shaker is energized by the right coil. For the voltage ratio measurements no calibration mass is attached to the right table. The test frequency is represented by $f$.

It is critical that the coil of the reciprocal transducer remain in the same static position in the magnetic gap so that the magnetic field to which the coil is exposed will not vary as a function of mass loading. This position must remain stable so that changes in coil current for different masses can be measured accurately. Temperature should remain constant so that the physical dimensions of the shaker, the transduction characteristics of the reciprocal transducer, and the resistance used for current measurements will remain constant. For greater stability, the coil is driven at about $20 \mathrm{~m} / \mathrm{s}^{2}\left(2 g_{n}\right)$ and allowed to remain energized throughout the measurements for all five masses. This results in measured voltages of typically $5 \mathrm{mV}$ to $500 \mathrm{mV}$. The two digital voltmeters are placed close to the moving element so that the connecting cables can be kept short to reduce electrical noise. A thermocouple was attached to the accelerometer and the temperature was stabilized to about $\pm 0.2^{\circ} \mathrm{C}$.

Figure 9 shows data for reciprocity and fringe-counting calibrations on a singleended accelerometer at $100 \mathrm{~Hz}$.

The estimated combined expanded uncertainty associated with the calibration results obtained by reciprocity at $100 \mathrm{~Hz}$ is $0.26 \%$ based on the current implementation of the measurement apparatus. This combined expanded uncertainty was calculated in accordance with methodologies described in the Guide to the Expression of Uncertainty in Measurement [6] using Type A and Type B evaluations of uncertainty components, including those contained in ISO documents on the calibration of vibration and shock transducers [7]. Using a coverage factor of 2, the estimated expanded relative uncertainty, $U$, at $100 \mathrm{~Hz}$ is: $0.5 \%$. 
For a set of ten calibrations by each method, the maximum difference between any two data points of either of the two methods was never more than $0.3 \%$, and the averages of the results obtained with the two methods agree within $0.04 \%$.

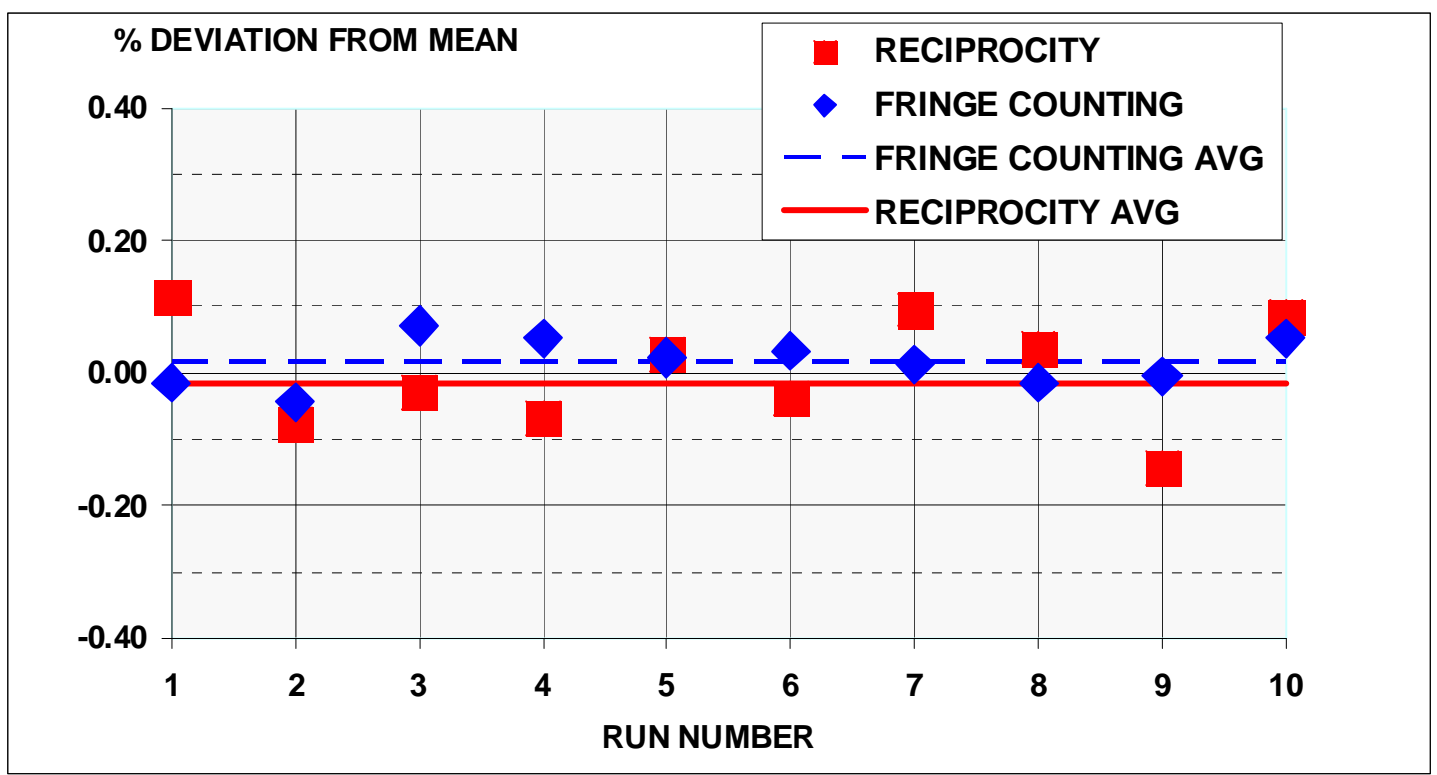

Figure 9. Data for reciprocity and fringe-counting calibrations on a singleended accelerometer at $100 \mathrm{~Hz}$

\section{Calibration by Laser Fringe-Counting Interferometry}

A flat mirror is mounted on the table located on the right side of the moving element opposite the table upon which the accelerometer is mounted (Fig.10). The shaker design allows optical access to the shaker mounting tables through a center hole in the magnet (figure 4). The acceleration, in meters per second squared, is given by

$$
A=\lambda v \pi^{2} f^{2} / 2,
$$

where $\lambda$ is the wavelength of He-Ne laser light in meters, $v$ is the number of fringe counts per vibration cycle, and $f$ is frequency in hertz $[2,8]$. To provide additional displacement, both the right and left coils can be driven in a push-pull configuration.

In addition to distortion and cross-axis motion which are critical to reciprocity calibrations, the critical elements of interferometer operation also include interferometer alignment and mechanical coupling between the shaker and the interferometer components. The shaker system was designed to minimize this coupling. The mass of the moving element (approximately $225 \mathrm{~g}$ ) is very small 
compared to the mass of the magnet (approximately $68 \mathrm{~kg}$ ) in order to minimize magnet movement. The shaker system is installed on an air isolation table to minimize the effects of floor movements. The magnets are moved into place on air-bearing guides, and during operation the magnets are locked into place by turning off the air to the bearings.

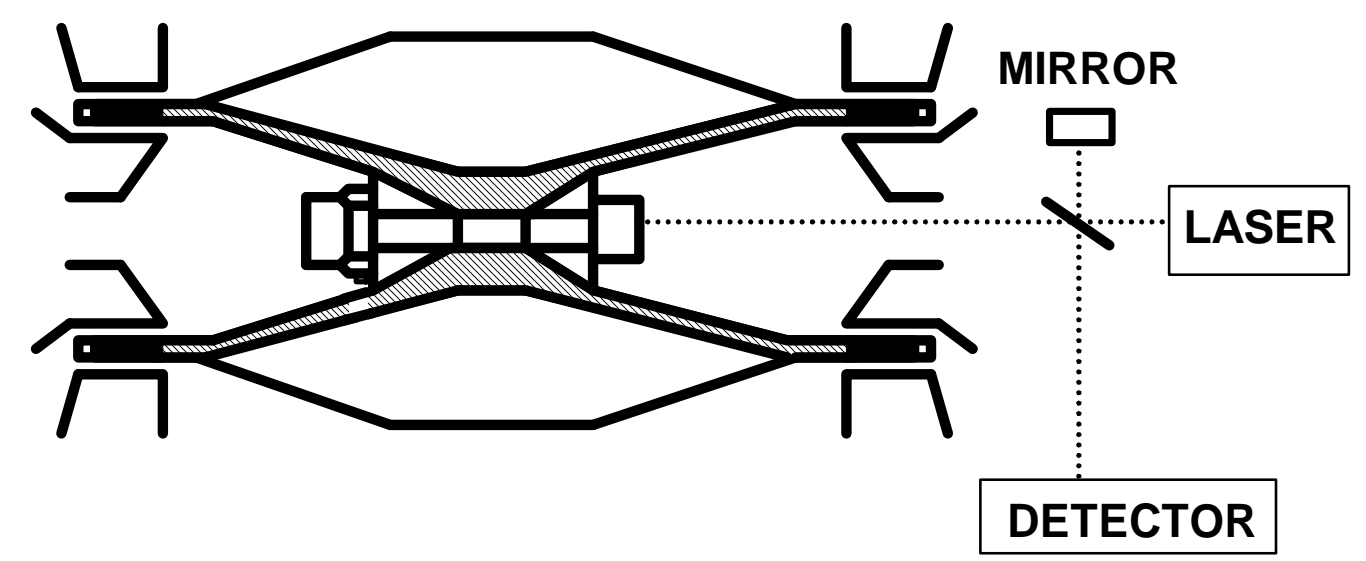

Figure 10. Schematic for fringe-counting interferometer setup on the Super Shaker

\section{Calibration by Minimum-Point Method Laser Interferometry}

The minimum-point method is based on the determination of displacement corresponding to the zero crossings of the Bessel function of the first kind and first order, $J_{1}$. This method utilizes the same setup as shown in Fig. 10 for calibrations performed in the frequency range of $1 \mathrm{kHz}$ to $5 \mathrm{kHz}$. In order to reject spectral components present in the output of the photo-detector except that corresponding to the fundamental frequency of vibration, the output of the photo-detector is filtered using a narrow band-pass filter centered at the fundamental frequency of vibration. The amplitude of vibration is then adjusted until the null corresponding to the desired zero crossing of $J_{1}$ is obtained. Peak displacement amplitudes of the first six zero crossings of $J_{1}$ for a He-Ne laser $(\lambda=632.8 \mathrm{~nm})$ are listed in Table 1. For minimum-point calibrations, at each tested frequency the number of the zero crossing was selected to obtain displacements corresponding to accelerations of approximately $50 \mathrm{~m} / \mathrm{s}^{2}$ or greater. 
Table 1. Peak Displacement Amplitudes for the First Six Zero Crossings

\begin{tabular}{|c|c|}
\hline Zero Crossing No. & $\begin{array}{c}\text { Displacement } \\
\text { (nm) }\end{array}$ \\
\hline 0 & 0.00 \\
\hline 1 & 192.95 \\
\hline 2 & 353.29 \\
\hline 3 & 512.31 \\
\hline 4 & 670.95 \\
\hline 5 & 829.42 \\
\hline
\end{tabular}

Once the displacement, $d$, is determined, the acceleration is calculated using the following equation:

$$
A=(2 \pi f)^{2} d .
$$

The sensitivity of the accelerometer is then obtained by dividing the accelerometer output voltage by the acceleration, $A$.

Calibration data using fringe-counting and minimum-point methods for a singleended accelerometer is shown in Fig. 11.

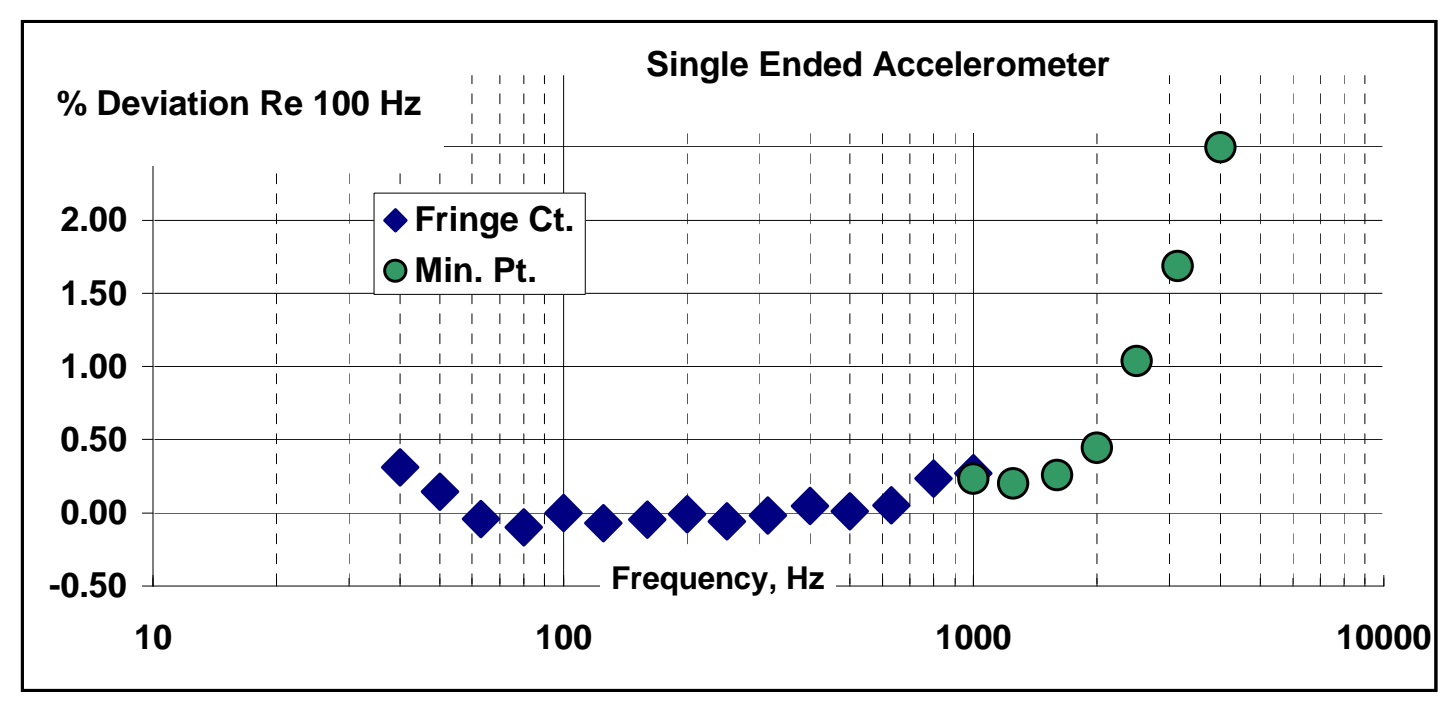

Figure 11. Calibration data for a single-ended accelerometer using fringe-counting and minimum-point methods

The estimated combined uncertainty associated with the calibration results obtained by minimum-point interferometry at $1 \mathrm{kHz}$ is $0.22 \%$ based on the current implementation of the measurement apparatus. This combined 
uncertainty was calculated in accordance with methodologies described in the Guide to the Expression of Uncertainty in Measurement [6] using Type A and Type B evaluations of uncertainty components, including those contained in ISO documents [7] on the calibration of vibration and shock transducers. Using a coverage factor of 2 , the estimated expanded relative uncertainty, $U$, for the minimum-point method at $1 \mathrm{kHz}$ is: $0.5 \%$, and the estimated expanded relative uncertainty, $U$, at $100 \mathrm{~Hz}$ is: $0.3 \%$ using fringe-counting. Data for the two calibration methods at the overlapping frequency of $1 \mathrm{kHz}$ agree to within less than $0.1 \%$.

\section{Calibration by Sine-Approximation Method Using Quadrature Laser Interferometry}

A piezo-electric shaker, as illustrated in figure 12, with a single mounting table and a driving element on the opposite end is typically used for accelerometer calibrations. The accelerometer is mounted at the center of the shaker table and mirrors are mounted around the perimeter of the accelerometer.

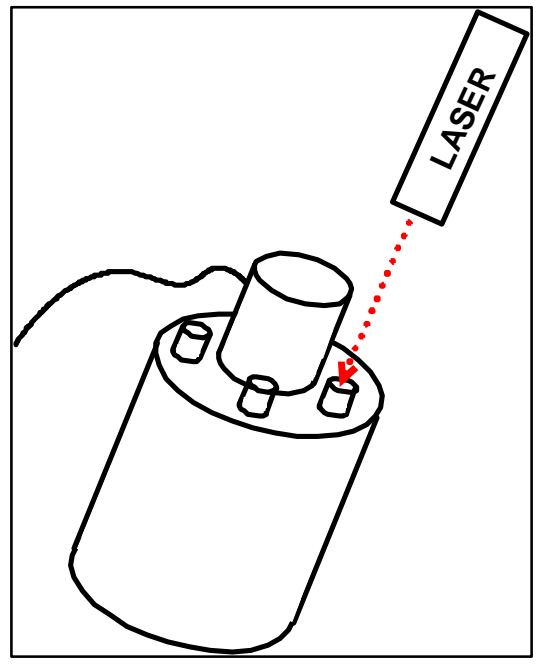

Figure 12. Conventional threemirror method for calibration using a single coil or piezoelectric shaker for interferometric calibration

Displacement must be measured at three or more positions and then averaged to get a good representation of the displacement of the plane on which the accelerometer is mounted.

Another approach is shown in Fig. 13 using the dual-coil Super Shaker. Here the accelerometer is mounted on the table opposite the reflecting mirror. Using reflection from the center of the mirror will enable accurate measurement of the displacement of the accelerometer using only the center position of the moving mirror provided the relative motion between the surfaces of the two tables is small. Tests conducted at NIST have shown that significant relative motion is experienced between tables fabricated from stainless steel or aluminum for 
frequencies higher than $5 \mathrm{kHz}$. Using a mounting table fabricated from beryllium with nickel plating provides for much smaller relative motion between the tables, and permits calibration of accelerometers up to $10,000 \mathrm{~Hz}$ using only one measuring point at the center of the mirror.

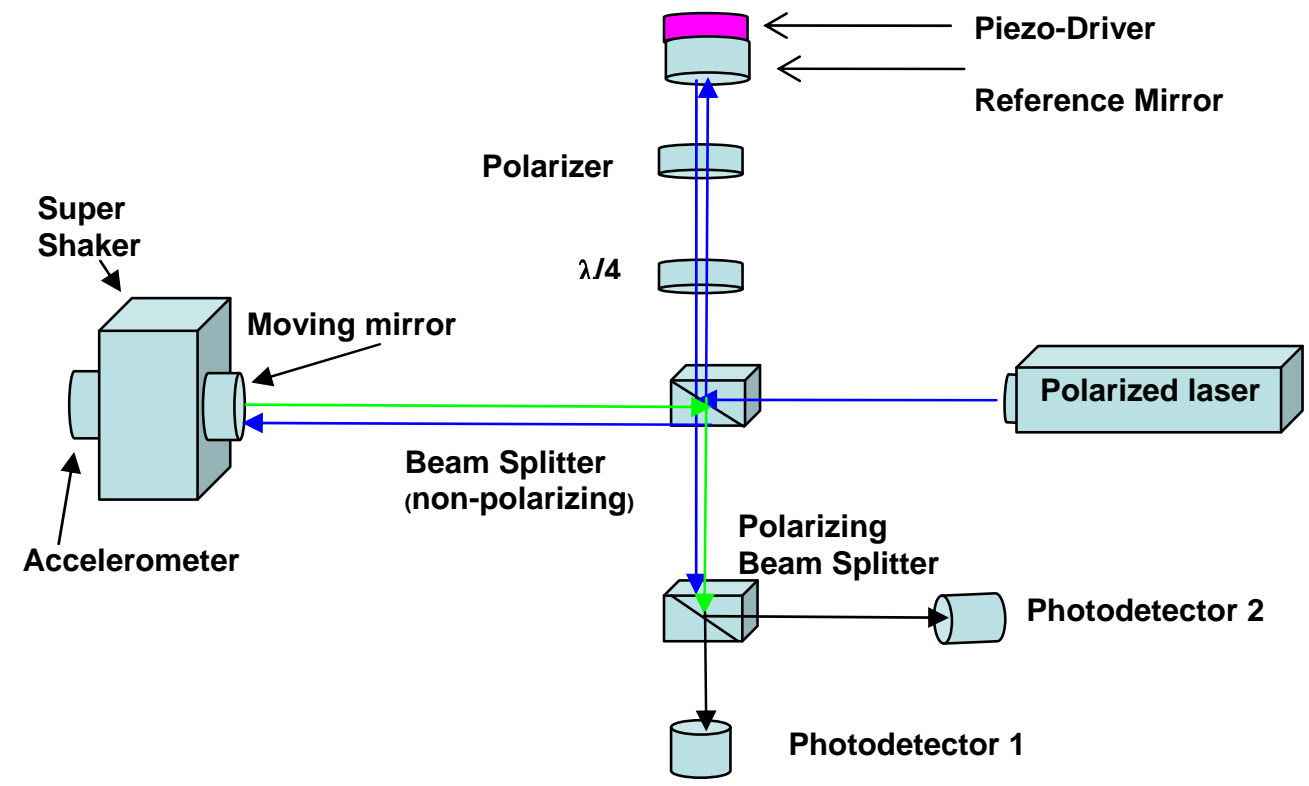

Figure 13. Setup using quadrature laser interferometer for sine-approximation, minimum-point, and fringe-counting methods

Using the setup shown in Fig. 13, calibration data can be obtained using the dual-coil shaker, for frequencies up to $1 \mathrm{kHz}$ using fringe-counting, and up to 5 $\mathrm{kHz}$ using the minimum-point method by monitoring the output from either detector 1 or detector 2 . These methods and procedures are documented in other NIST publications [9] and in ISO 16063 - Part 11 [7]. In order to calibrate at frequencies higher than $5 \mathrm{kHz}$, the sine-approximation method is used. This method is useful at higher frequencies where calibrations at smaller displacements must be made due to amplitude limitations in electro-dynamic shakers. The current implementation of the NIST dual-coil shaker limits the acceleration to approximately $200 \mathrm{~m} / \mathrm{s}^{2}$. In actual practice the sine-approximation method gives good results over a wide frequency range, $100 \mathrm{~Hz}$ to $10,000 \mathrm{~Hz}$. It is also much less demanding on the shaker, since it can operate at lower accelerations than the minimum-point method for higher frequencies. Typically, 
the sine-approximation method is used at NIST for calibration of accelerometers at accelerations of $200 \mathrm{~m} / \mathrm{s}^{2}$ to $500 \mathrm{~m} / \mathrm{s}^{2}$ from $100 \mathrm{~Hz}$ to $10 \mathrm{kHz}$, and fringecounting is used for frequencies of $100 \mathrm{~Hz}$ and below. This range of acceleration is used to minimize the heating effects on the shaker. The minimum-point method can be used as check on the other methods, but is not usually used at NIST on a daily basis.

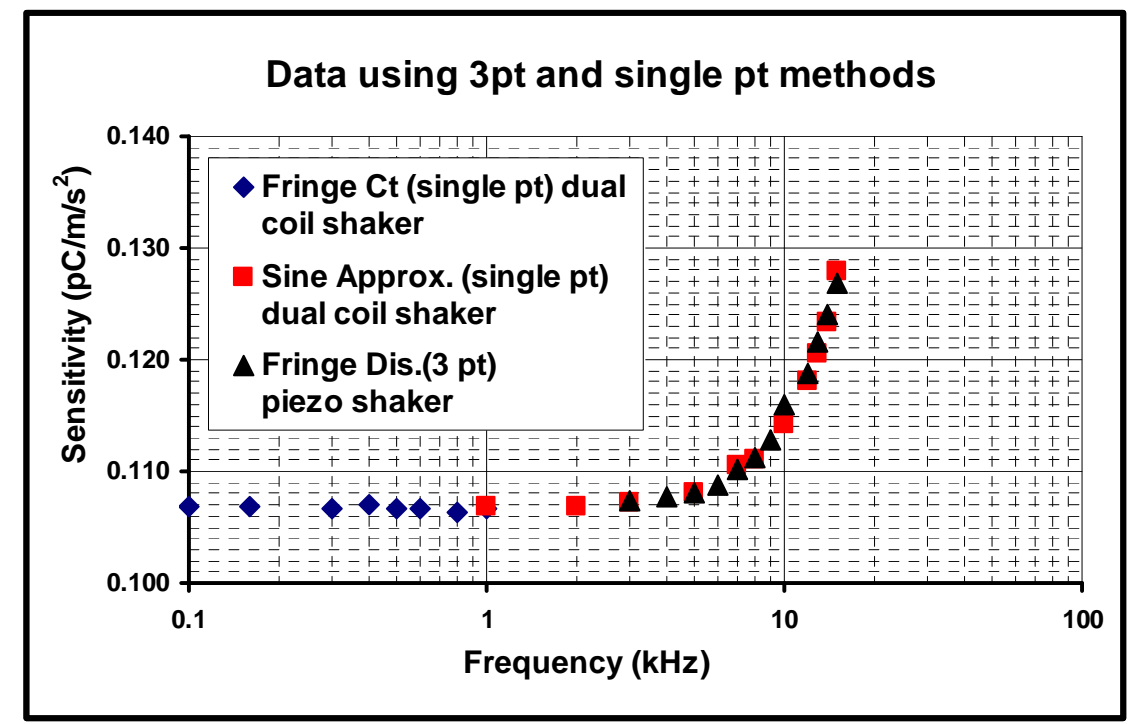

Figure 14. Calibration data on a single-ended accelerometer using single point (SS2 shaker) and three point (piezo-electric shaker) techniques

Calibration data using fringe-counting and sine-approximation methods for a single-ended accelerometer on SS2 is shown in Fig. 14. This calibration uses a single point measurement, and thereby requires minimal adjustment to the interferometer. Data using fringe-disappearance on a piezo-electric shaker is also shown in Fig. 14. This data requires measurements at three positions on the shaker and thus requires realignment for each position.

The combined relative uncertainties for both the fringe-counting and sineapproximation methods were calculated in accordance with methodologies described in the Guide to the Expression of Uncertainty in Measurement [6] using Type A and Type B evaluations of uncertainty components, including those contained in ISO documents [7] on the calibration of vibration and shock transducers. For the fringe-counting method, using a coverage factor of 2 , the estimated expanded uncertainty, $U$, at $100 \mathrm{~Hz}$ is $0.5 \%$. For the sineapproximation method, again using a coverage factor of 2, the estimated expanded relative uncertainty, $U$, at $1 \mathrm{kHz}$ is $0.5 \%$, at $5 \mathrm{kHz}$ is $0.75 \%$, and at 10 $\mathrm{kHz}$ is $1 \%$ for the NIST Super Shaker. 


\section{Summary of Uncertainties}

The overall uncertainties of the two shakers were calculated as stated above using the ISO 16063-11 standard [7]. Figure 15 gives the results of these calculations for the fringe-counting, minimum-point, and sine-approximation methods. The estimated expanded uncertainties, $U$, for frequencies $40 \mathrm{~Hz}$ to $100 \mathrm{~Hz}$ were found to be less than $0.5 \%$ using fringe-counting. These results are published in BIPM/CIPM key comparison CCAUV.V-K1 (Vibration), 2001 [10] for SS1. Additional details on the uncertainty calculations are given in the Appendix of this document.

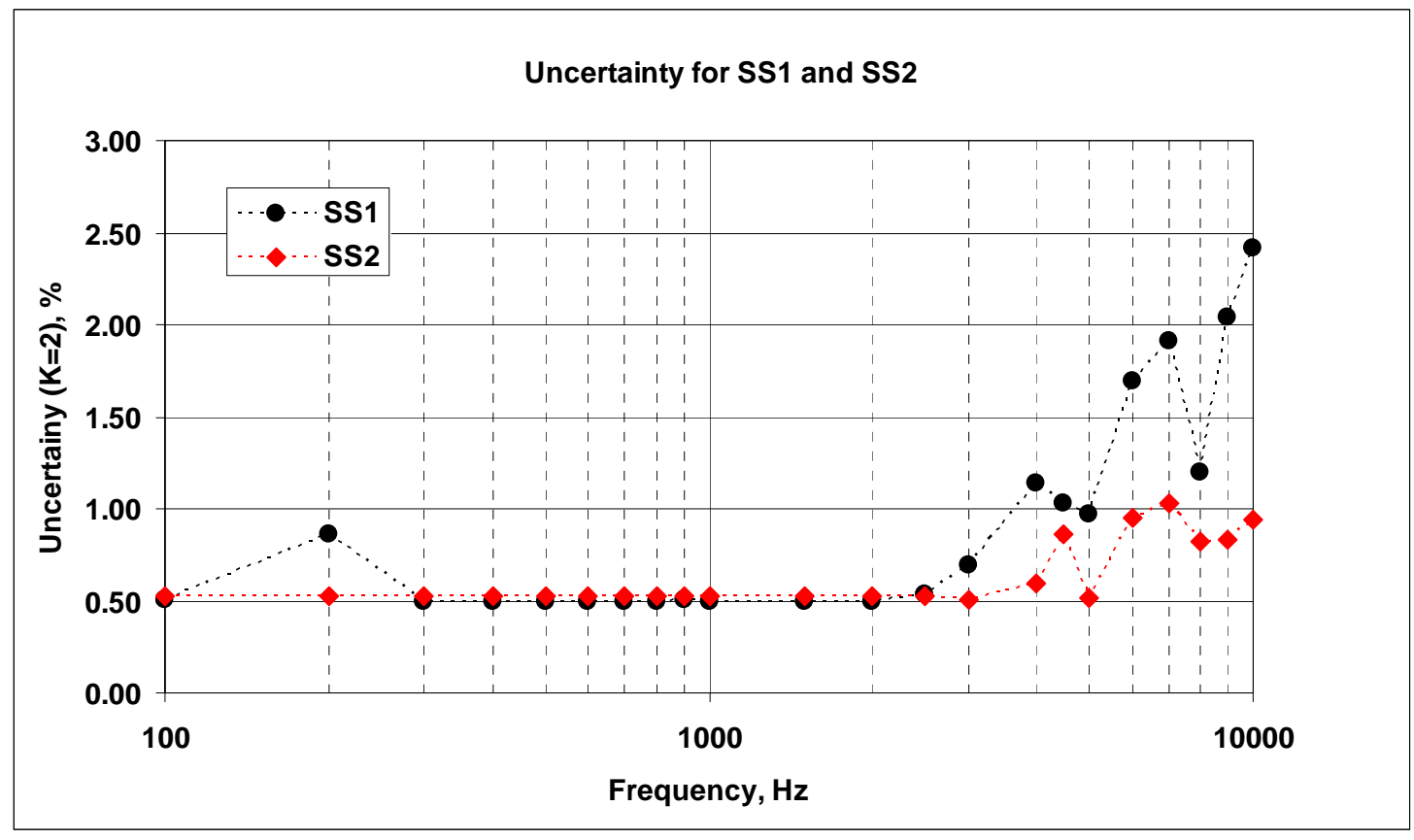

Figure 15. Calculated uncertainties for SS1 and SS2

\section{Performance}

The two shakers SS1 (AFMETCAL) and SS2 (NIST) were used to calibrate a single-ended accelerometer. The results, given in Fig. 16, show good agreement between the two systems and are within the uncertainty estimates. 


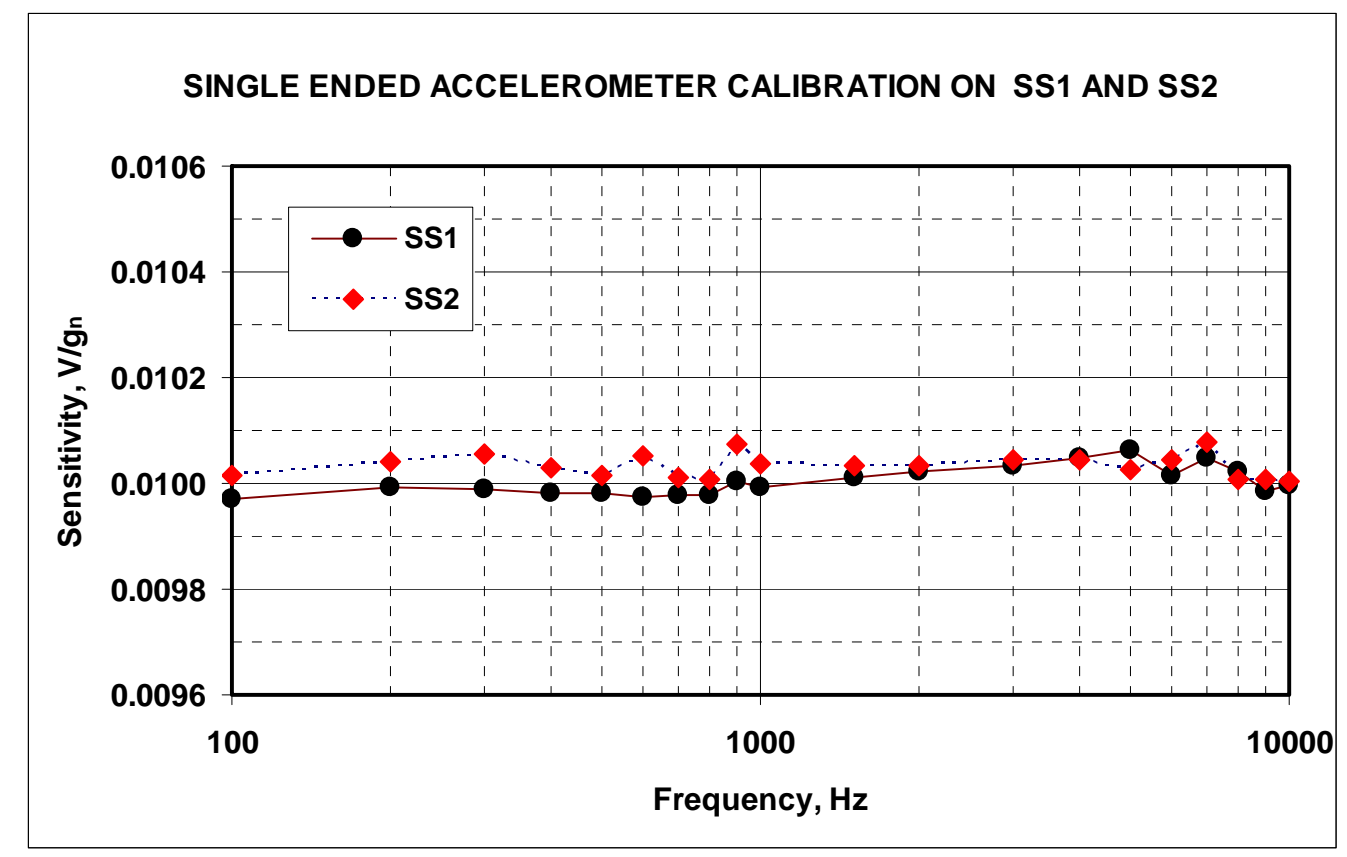

Figure 16. Calibration results of a single-ended accelerometer on NIST SS2 and AFMETCAL SS1

\section{Summary}

A dual-coil electrodynamic shaker has been developed for improved accelerometer calibration by both reciprocity and interferometric methods. The shaker uses a uniquely designed aluminum moving element with two mounting tables and a damped flexure support system for the moving element. The shaker has very low harmonic distortion and low cross motion over most of the operating range. Test results show that the shaker has a useful range of $30 \mathrm{~Hz}$ to $10 \mathrm{kHz}$ for primary calibrations.

The shaker can utilize all four primary methods for accelerometer calibrations: reciprocity, fringe-counting, minimum-point, and sine-approximation. Reciprocity calibration is limited to frequencies of $1 \mathrm{kHz}$ or less, because of inductive interaction between the two coils, unless special procedures are used to cancel these effects. Fringe-counting is used from $30 \mathrm{~Hz}$ to $1 \mathrm{kHz}$. The minimum-point method is used from about $100 \mathrm{~Hz}$ to $5 \mathrm{kHz}$. The upper limit is set by the upper acceleration limit of the shaker, which is about $200 \mathrm{~m} / \mathrm{s}^{2}$ at the higher frequencies. Sine-approximation is used from $100 \mathrm{~Hz}$ to $10 \mathrm{kHz}$.

Calibrations on NIST's SS2 have estimated uncertainties of $0.5 \%$ to $1.0 \%$ over the frequency range $30 \mathrm{~Hz}$ to $10 \mathrm{kHz}$. SS1 (AFMETCAL) can obtain calibrations with estimated uncertainties of $0.5 \%$ to $2.4 \%$ over the frequency range of $30 \mathrm{~Hz}$ to $10 \mathrm{kHz}$. SS2 at NIST uses softer flexures for the moving element, which may 
provide smaller relative motion between the two mounting tables. However the specific cause of the difference in uncertainties is not presently known.

\section{References}

1. Payne B. F., Booth G. B., "The NIST Super Shaker Project," Proc. Metrologie 95, 1995, 296-301.

2. Payne, B., Evans, D., "Comparison of Results of Calibrating the Magnitude of the Sensitivity of Accelerometers by Laser Interferometry and Reciprocity," Metrologia, 1999, 36, 391-394.

3. S. Levy and R.R. Bouche, Calibration of Vibration Pickups by the Reciprocity Method, J. Research NBS 57, 227-243 (1956) RP2714.

4. D.C. Robinson, M.R. Serbyn, B. F. Payne, A Description of NBS Calibration Services in Mechanical Vibration and Shock, NBS Technical Note 1232, 1987.

5. Payne, B., Evans, D., "Primary Calibration of Accelerometers at NIST Using a Dual Coil Shaker," Proceedings of the $72^{\text {nd }}$ Shock and Vibration Symposium, Nov 12-16, 2001, Destin, FL, pp. 138-142.

6. "Guide to the Expression of Uncertainty in Measurement", $2^{\text {nd }} E d$. ., International Organization for Standardization, Switzerland, 1995, ISBN 9210188-9

7. International Standard ISO 16063-11, Primary vibration calibrations by laser interferometry, International Organization for Standardization, Geneva, 1999.

8. Payne, B., Harper, K., "Improvements in Accelerometer Calibration Using Fringe-Counting and Minimum-point Methods," Proceedings of the $74^{\text {th }}$ Shock and Vibration Symposium, Nov. 12-16, 2001, Destin, FL, pp. 138-142.

9. Payne, B. "Single Point Optical Calibration of Accelerometers at NIST," Seventh International Conference on Vibration Measurements by Laser Techniques: Advances and Applications, Proc. of SPIE Vol. 6345, 634504, (2006).

10. BIPM/CIPM key comparison CCAUV.V-K1 (Vibration), 2001 
Appendix: Super Shaker Characterization 


\begin{tabular}{|rrr|}
\hline $\begin{array}{r}\text { Frequency } \\
\mathbf{( H z )}\end{array}$ & $\begin{array}{r}\text { Distortion at 2g } \\
\mathbf{( \% )}\end{array}$ & $\begin{array}{r}\text { Distortion at 3g } \\
\mathbf{( \% )}\end{array}$ \\
25 & & \\
30 & 0.63 & \\
40 & 0.42 & \\
50 & 0.34 & 0.51 \\
80 & & 0.44 \\
400 & & 0.41 \\
160 & & 0.46 \\
200 & & 0.3 \\
300 & & 0.43 \\
400 & & 0.44 \\
500 & & 0.61 \\
600 & & 0.66 \\
700 & & 0.65 \\
800 & & 0.4 \\
900 & & 0.17 \\
1000 & & \\
1500 & 0.68 & 0.12 \\
2000 & & 0.25 \\
3000 & & 0.17 \\
4000 & & 0.19 \\
5000 & & 0.16 \\
6000 & 0.45 \\
7000 & 0.06 \\
8000 & 0.08 \\
9000 & 0.42 \\
10000 & &
\end{tabular}




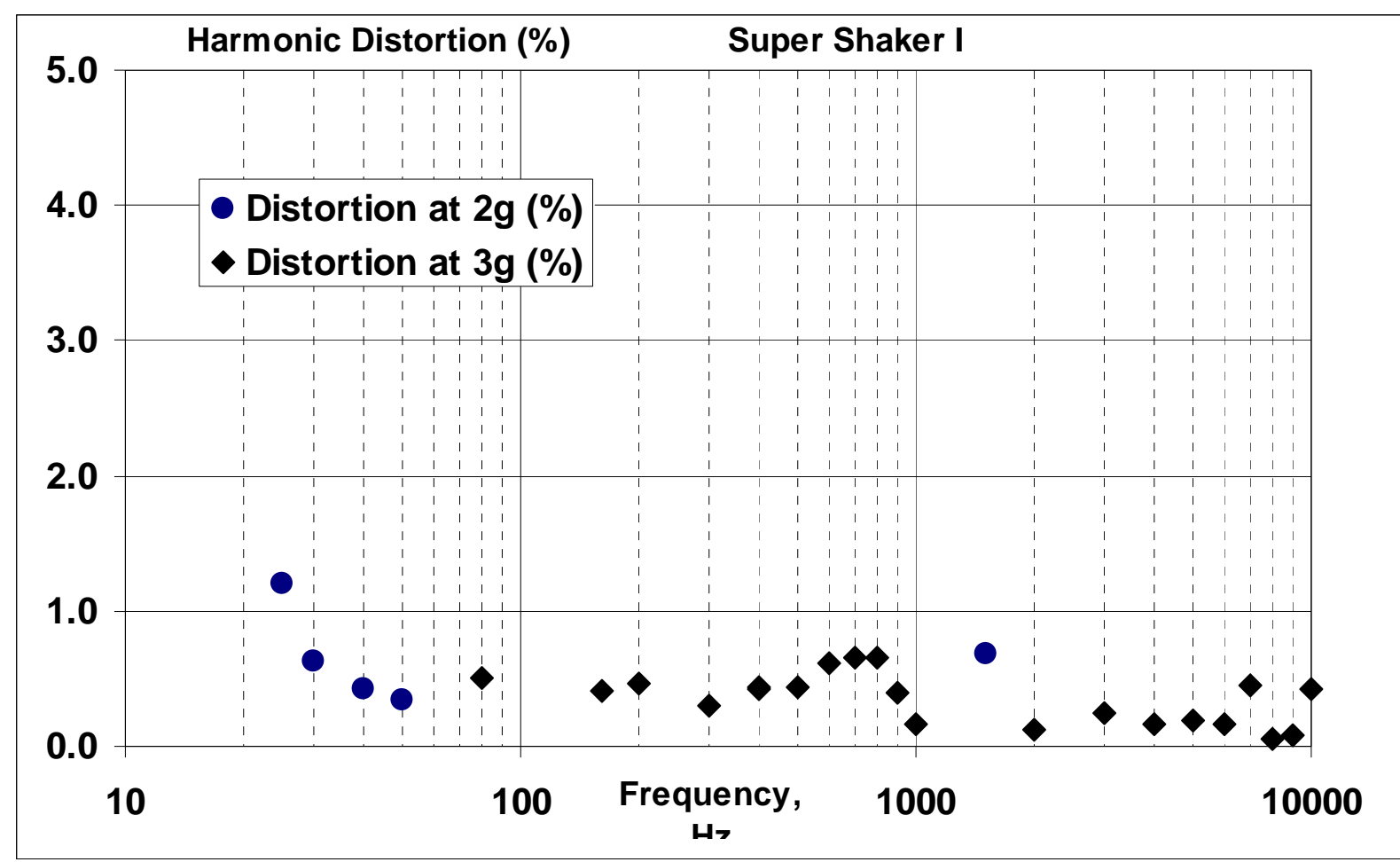




\begin{tabular}{|c|c|c|c|c|c|c|c|}
\hline \multicolumn{2}{|c|}{ Super Shaker I } & \multicolumn{3}{|c|}{ Cross Axis Motion } & \multicolumn{2}{|c|}{ August 2008} & \\
\hline Frequency $(\mathrm{Hz})$ & $\mathrm{X}$ axis $(\mathrm{mV})$ & $z$ axis(mV) & y axis(mV) & $x / z \%$ & $y / z \%$ & $\begin{array}{l}\text { RSS Total } \\
\text { Cross Axis }\end{array}$ & $\begin{array}{l}\text { \% Uncert } \\
\text { Cross Axis }\end{array}$ \\
\hline 40 & 23.90 & 758.50 & 5.50 & $3.2 \%$ & $0.73 \%$ & $3.2 \%$ & 0.06 \\
\hline 50 & 23.00 & 684.10 & 6.10 & $3.4 \%$ & $0.89 \%$ & $3.5 \%$ & 0.07 \\
\hline 80 & 22.40 & 616.20 & 5.90 & $3.6 \%$ & $0.96 \%$ & $3.8 \%$ & 0.08 \\
\hline 100 & 46.00 & 1136.00 & 12.60 & $4.0 \%$ & $1.11 \%$ & $4.2 \%$ & 0.08 \\
\hline 200 & 99.10 & 1071.00 & 231.70 & $9.3 \%$ & $21.63 \%$ & $23.5 \%$ & 0.47 \\
\hline 300 & 17.10 & 1035.00 & 19.70 & $1.7 \%$ & $1.90 \%$ & $2.5 \%$ & 0.05 \\
\hline 400 & 14.00 & 1041.00 & 11.00 & $1.3 \%$ & $1.06 \%$ & $1.7 \%$ & 0.03 \\
\hline 500 & 6.00 & 570.00 & & $1.1 \%$ & $0.00 \%$ & $1.1 \%$ & 0.02 \\
\hline 600 & 0.60 & 705.00 & 16.20 & $0.1 \%$ & $2.30 \%$ & $2.3 \%$ & 0.05 \\
\hline 700 & 16.60 & 706.00 & 9.10 & $2.4 \%$ & $1.29 \%$ & $2.7 \%$ & 0.05 \\
\hline 800 & 1.20 & 702.00 & 14.00 & $0.2 \%$ & $1.99 \%$ & $2.0 \%$ & 0.04 \\
\hline 900 & 5.50 & 970.40 & 32.70 & $0.6 \%$ & $3.37 \%$ & $3.4 \%$ & 0.07 \\
\hline 1000 & 0.18 & 709.00 & 23.00 & $0.0 \%$ & $3.24 \%$ & $3.2 \%$ & 0.06 \\
\hline 1500 & 10.20 & 966.00 & 17.60 & $1.1 \%$ & $1.82 \%$ & $2.1 \%$ & 0.04 \\
\hline 2000 & 10.70 & 699.50 & 17.80 & $1.5 \%$ & $2.54 \%$ & $3.0 \%$ & 0.06 \\
\hline 2500 & 53.00 & 1061.00 & 29.60 & $5.0 \%$ & $2.79 \%$ & $5.7 \%$ & 0.11 \\
\hline 3000 & 14.70 & 672.80 & 71.40 & $2.2 \%$ & $10.61 \%$ & $10.8 \%$ & 0.22 \\
\hline 3500 & 125.30 & 1003.00 & 144.90 & $12.5 \%$ & $14.45 \%$ & $19.1 \%$ & 0.38 \\
\hline 4000 & 54.30 & 650.90 & 25.30 & $8.3 \%$ & $3.89 \%$ & $9.2 \%$ & 0.18 \\
\hline 4500 & 53.70 & 967.40 & 134.60 & $5.6 \%$ & 13.91\% & $15.0 \%$ & 0.30 \\
\hline 5000 & 23.70 & 643.50 & 54.70 & $3.7 \%$ & $8.50 \%$ & $9.3 \%$ & 0.19 \\
\hline 5500 & 39.90 & 964.60 & 66.80 & $4.1 \%$ & $6.93 \%$ & $8.1 \%$ & 0.16 \\
\hline 6000 & 51.10 & 641.70 & 10.70 & $8.0 \%$ & $1.67 \%$ & $8.1 \%$ & 0.16 \\
\hline 6500 & 82.20 & 931.80 & 35.60 & $8.8 \%$ & $3.82 \%$ & $9.6 \%$ & 0.19 \\
\hline 7000 & 27.70 & 606.10 & 77.60 & $4.6 \%$ & $12.80 \%$ & $13.6 \%$ & 0.27 \\
\hline 7500 & 266.10 & 966.00 & 2.80 & $27.5 \%$ & $0.29 \%$ & $27.5 \%$ & 0.55 \\
\hline 8000 & 267.30 & 793.90 & 31.70 & $33.7 \%$ & $3.99 \%$ & $33.9 \%$ & 0.68 \\
\hline 8500 & 224.00 & 814.90 & 44.70 & $27.5 \%$ & $5.49 \%$ & $28.0 \%$ & 0.56 \\
\hline 9000 & 46.10 & 700.00 & 266.70 & $6.6 \%$ & $38.10 \%$ & $38.7 \%$ & 0.77 \\
\hline 9500 & 238.40 & 725.40 & 67.90 & $32.9 \%$ & $9.36 \%$ & $34.2 \%$ & 0.68 \\
\hline 10000 & 252.00 & 610.80 & 60.00 & $41.3 \%$ & $9.82 \%$ & $42.4 \%$ & 0.64 \\
\hline
\end{tabular}




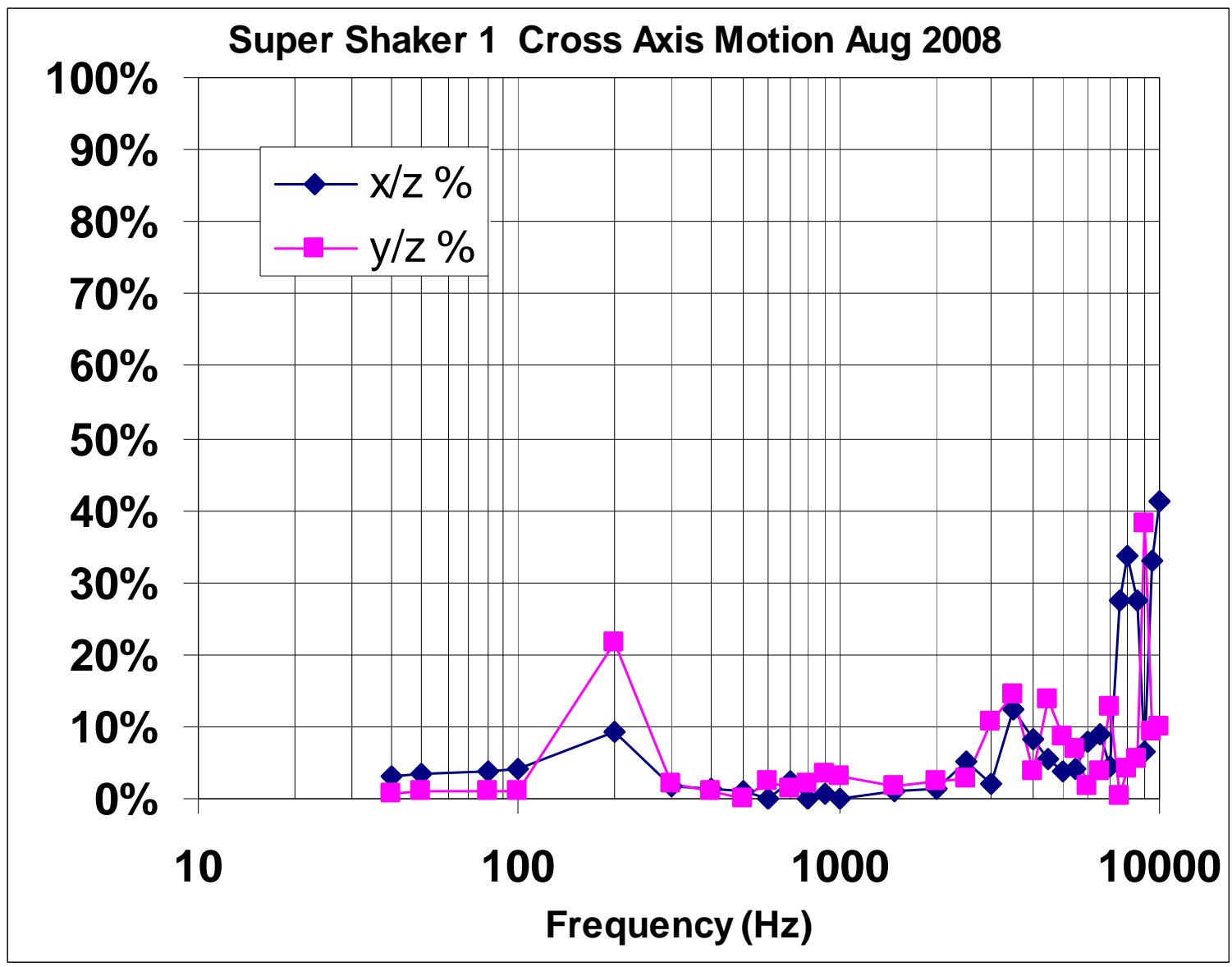


Super Shaker I $\quad$ Relative Motion $\quad$ September 2009

Freq (Hz) VIB Pos* 1 (nm) QLI Pos 1 (nm) \% diff. VIB Pos 2 (nm) QLI Pos 2 (nm) \% diff. VIB Pos 3 (nm) QLI Pos 3 (nm) \% diff. AVG

\begin{tabular}{|c|c|c|c|c|c|c|c|c|c|c|}
\hline 500 & 5890.0 & 5875.0 & -0.3 & 5912.0 & 5892.0 & -0.3 & 6939.0 & 6933.0 & -0.1 & -0.23 \\
\hline 1000 & 1254.0 & 1255.0 & 0.1 & 1472.0 & 1476.0 & 0.3 & 1758.0 & 1749.0 & -0.5 & -0.05 \\
\hline 2000 & 341.6 & 341.1 & -0.1 & 364.0 & 363.8 & -0.1 & 442.9 & 443.5 & 0.1 & -0.02 \\
\hline 3000 & 175.3 & 175.0 & -0.2 & 162.0 & 162.2 & 0.1 & 193.2 & 194.4 & 0.6 & 0.19 \\
\hline 4000 & 102.5 & 102.6 & 0.1 & 88.3 & 89.4 & 0.7 & 106.3 & 107.0 & 0.7 & 0.485 \\
\hline 5000 & 63.6 & 64.0 & 0.6 & 66.3 & 66.7 & 0.5 & 66.4 & 66.4 & 0.0 & 0.3 \\
\hline 6000 & 46.4 & 47.0 & 1.1 & 45.2 & 45.7 & 1.0 & 46.4 & 46.5 & 0.3 & 0.83 \\
\hline 7000 & 32.9 & 33.2 & 0.8 & 32.5 & 32.9 & 1.1 & 32.7 & 32.9 & 0.73 & 0.9 \\
\hline 8000 & 26.3 & 26.4 & 0.3 & 26.0 & 26.1 & 0.6 & 26.4 & 26.3 & -0.3 & 0.21 \\
\hline 9000 & 20.1 & 20.4 & 1.5 & 20.2 & 20.3 & 0.7 & 20.1 & 20.2 & 0.2 & 0.79 \\
\hline .0000 & 16.1 & 16.5 & 2.3 & 16.4 & 16.3 & -0.1 & 16.2 & 16.4 & 0.7 & 0.99 \\
\hline
\end{tabular}

*VIB Pos = location (on mirror mounted on first mounting table) off of which the vibrometer laser was reflecting; similarly QLI Pos = location (on mirror mounted on second mounting table) off of which the laser from the quadrature interferometer was reflecting. Each system measured three points about the center sequentially. These were averaged to yield an estimate of the motion of the center of each mirror. 


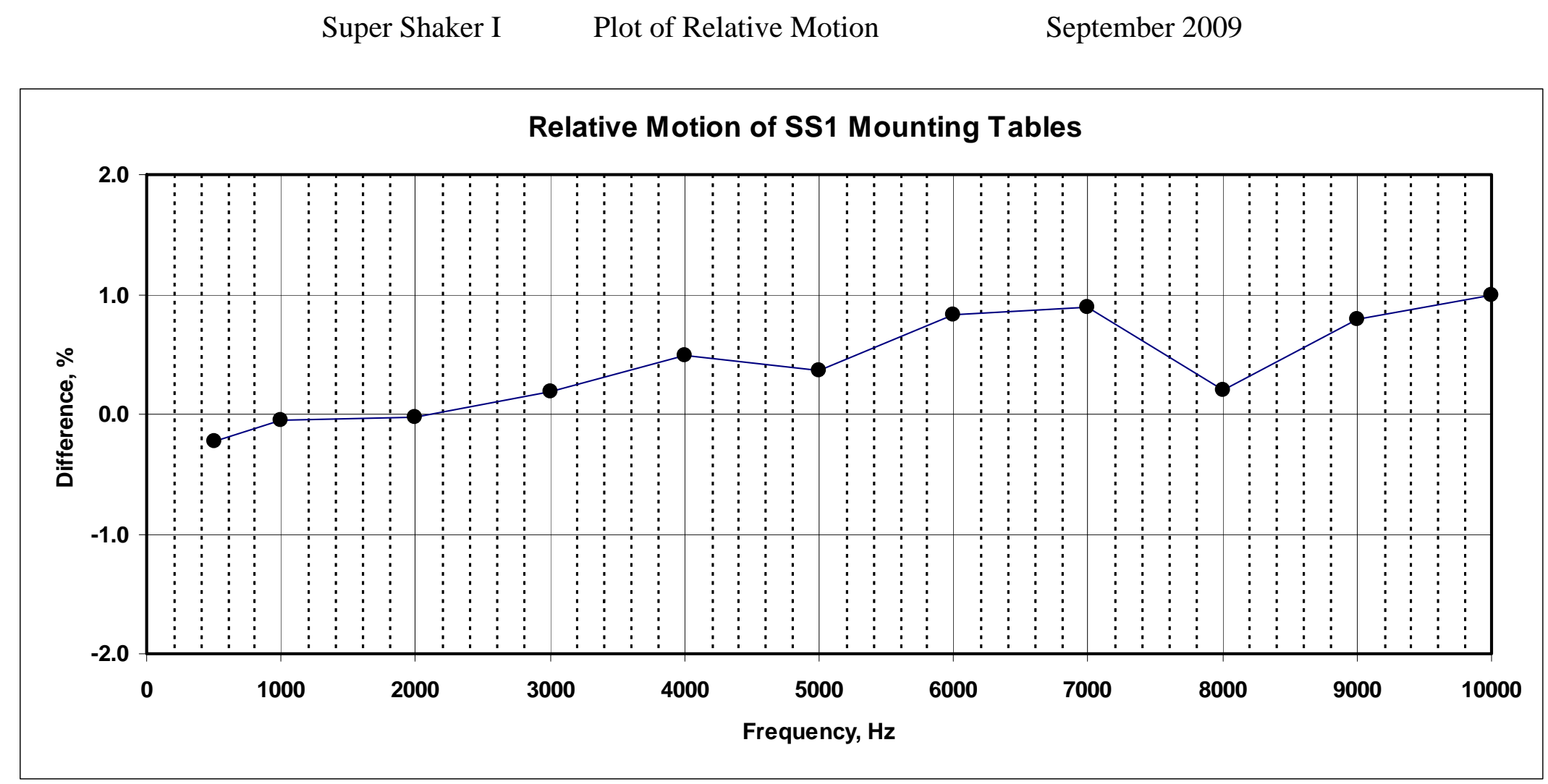


Super Shaker I Repeatability Tests

Repeatability Test SS1 Both coils in use; acceleration amplitude: 2-3g AF accelerometer May 282009

Repeatability Test SS1 Both coils in use; acceleration amplitude: 2-3g AF accelerometer May 282009

\begin{tabular}{|c|c|c|c|c|c|c|c|c|c|}
\hline Freq & S(run1) & S(run2) & S(run3) & Mean & Run1_Run2 \% & Run1_Run3 \% & Run2_Run3 \% & Prob. Error & Prob Error(\%) \\
\hline 100 & 0.009963 & 0.009994 & 0.010032 & 0.01000 & -0.3 & -0.7 & -0.4 & 1.35E-05 & 0.13 \\
\hline 200 & 0.009973 & 0.010001 & 0.010018 & 0.01000 & -0.3 & -0.5 & -0.2 & 8.85E-06 & 0.09 \\
\hline 300 & 0.009972 & 0.009994 & 0.010026 & 0.01000 & -0.2 & -0.5 & -0.3 & 1.06E-05 & 0.11 \\
\hline 400 & 0.009983 & 0.009989 & 0.010027 & 0.01000 & -0.1 & -0.4 & -0.4 & $9.29 \mathrm{E}-06$ & 0.09 \\
\hline 500 & 0.010013 & 0.010001 & 0.009973 & 0.01000 & 0.1 & 0.4 & 0.3 & 7.99E-06 & 0.08 \\
\hline 600 & 0.010034 & 0.010003 & 0.010015 & 0.01002 & 0.3 & 0.2 & -0.1 & 6.09E-06 & 0.06 \\
\hline 700 & 0.009999 & 0.01001 & 0.009967 & 0.00999 & -0.1 & 0.3 & 0.4 & 8.70E-06 & 0.09 \\
\hline 800 & 0.010016 & 0.010009 & 0.010022 & 0.01002 & 0.1 & -0.1 & -0.1 & $2.53 \mathrm{E}-06$ & 0.03 \\
\hline 900 & 0.010022 & 0.01003 & 0.010029 & 0.01003 & -0.1 & -0.1 & 0.0 & 1.70E-06 & 0.02 \\
\hline 1000 & 0.009999 & 0.010026 & 0.010003 & 0.01001 & -0.3 & 0.0 & 0.2 & $5.67 \mathrm{E}-06$ & 0.06 \\
\hline 1500 & 0.010028 & 0.010033 & 0.010021 & 0.01003 & 0.0 & 0.1 & 0.1 & $2.35 \mathrm{E}-06$ & 0.02 \\
\hline 2000 & 0.010029 & 0.010057 & 0.010014 & 0.01003 & -0.3 & 0.1 & 0.4 & 8.50E-06 & 0.08 \\
\hline 2500 & 0.010047 & 0.010068 & 0.010077 & 0.01006 & -0.2 & -0.3 & -0.1 & $6.00 \mathrm{E}-06$ & 0.06 \\
\hline 3000 & 0.010035 & 0.010056 & 0.010024 & 0.01004 & -0.2 & 0.1 & 0.3 & 6.33E-06 & 0.06 \\
\hline 4000 & 0.010062 & 0.010082 & 0.010094 & 0.01008 & -0.2 & -0.3 & -0.1 & 6.30E-06 & 0.06 \\
\hline 4500 & 0.01005 & 0.010073 & 0.010046 & 0.01006 & -0.2 & 0.0 & 0.3 & 5.67E-06 & 0.06 \\
\hline 5000 & 0.010086 & 0.010106 & 0.010078 & 0.01009 & -0.2 & 0.1 & 0.3 & $5.62 \mathrm{E}-06$ & 0.06 \\
\hline 5500 & 0.010098 & 0.01011 & 0.010106 & 0.01010 & -0.1 & -0.1 & 0.0 & 2.38E-06 & 0.02 \\
\hline 6000 & 0.010101 & 0.010142 & 0.010128 & 0.01012 & -0.4 & -0.3 & 0.1 & 8.12E-06 & 0.08 \\
\hline 6500 & 0.010053 & 0.010098 & 0.010071 & 0.01007 & -0.4 & -0.2 & 0.3 & 8.82E-06 & 0.09 \\
\hline 7000 & 0.010064 & 0.010133 & 0.010083 & 0.01009 & -0.7 & -0.2 & 0.5 & 1.39E-05 & 0.14 \\
\hline 7500 & 0.010179 & 0.010182 & 0.010157 & 0.01017 & 0.0 & 0.2 & 0.2 & 5.32E-06 & 0.05 \\
\hline 8000 & 0.010138 & 0.010204 & 0.010205 & 0.01018 & -0.6 & -0.7 & 0.0 & 1.50E-05 & 0.15 \\
\hline 8500 & 0.010212 & 0.010204 & 0.010164 & 0.01019 & 0.1 & 0.5 & 0.4 & 1.00E-05 & 0.10 \\
\hline 9000 & 0.010196 & 0.010234 & 0.010231 & 0.01022 & -0.4 & -0.3 & 0.0 & 8.23E-06 & 0.08 \\
\hline 10000 & 0.010224 & 0.01025 & 0.010194 & 0.01022 & -0.3 & 0.3 & 0.5 & 1.09E-05 & 0.11 \\
\hline
\end{tabular}

Probable Error of the mean calculated according to CRC "Standard Mathematical Tables" 


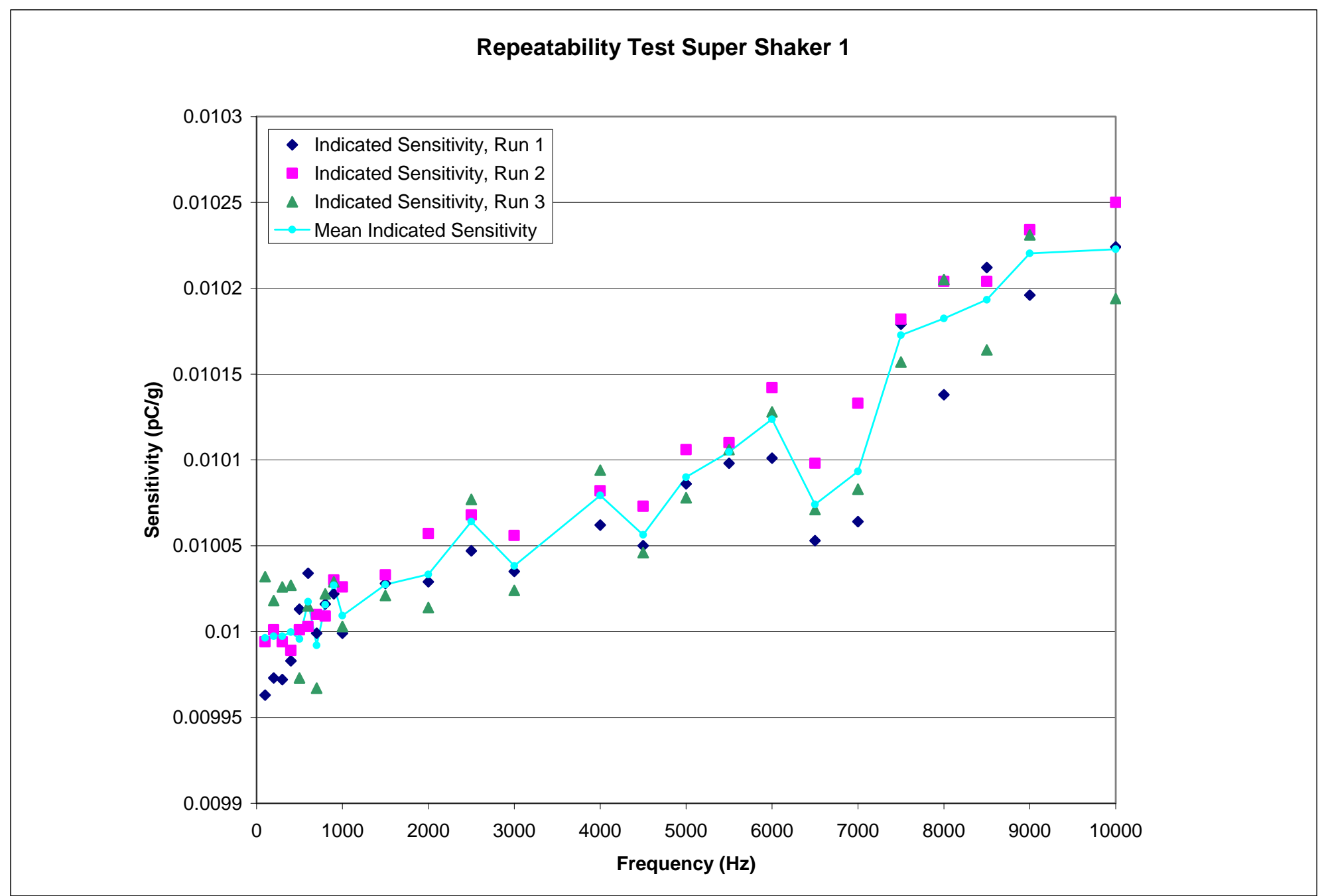




\section{Super Shakers I and II Uncertainty Estimates $\quad$ August 2010}

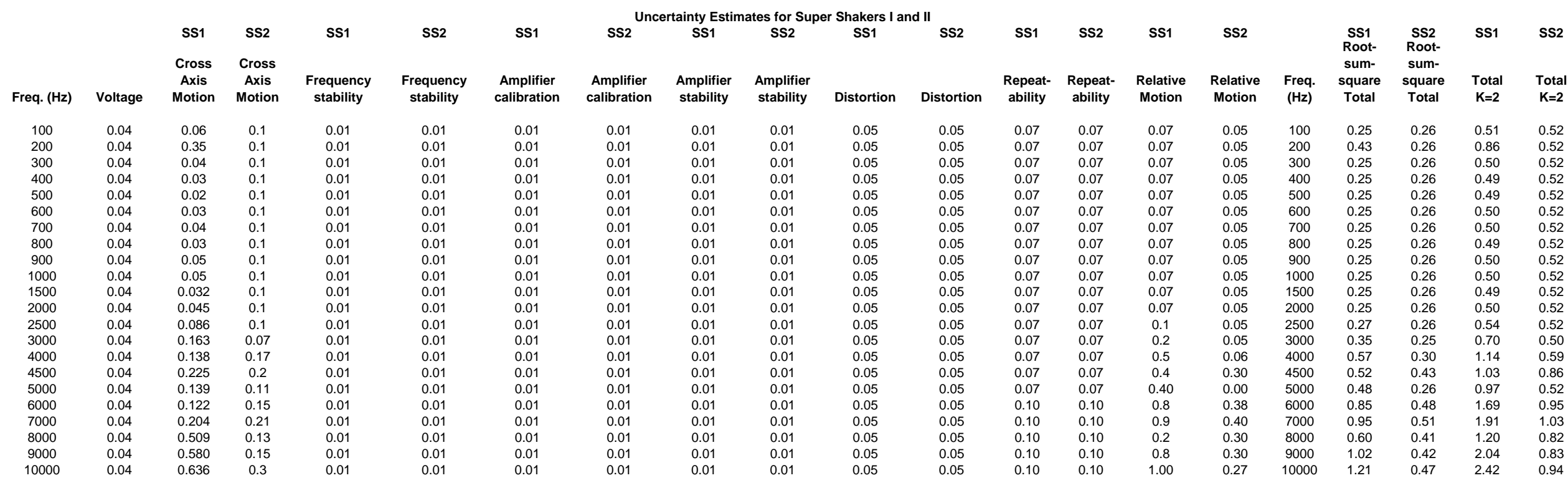




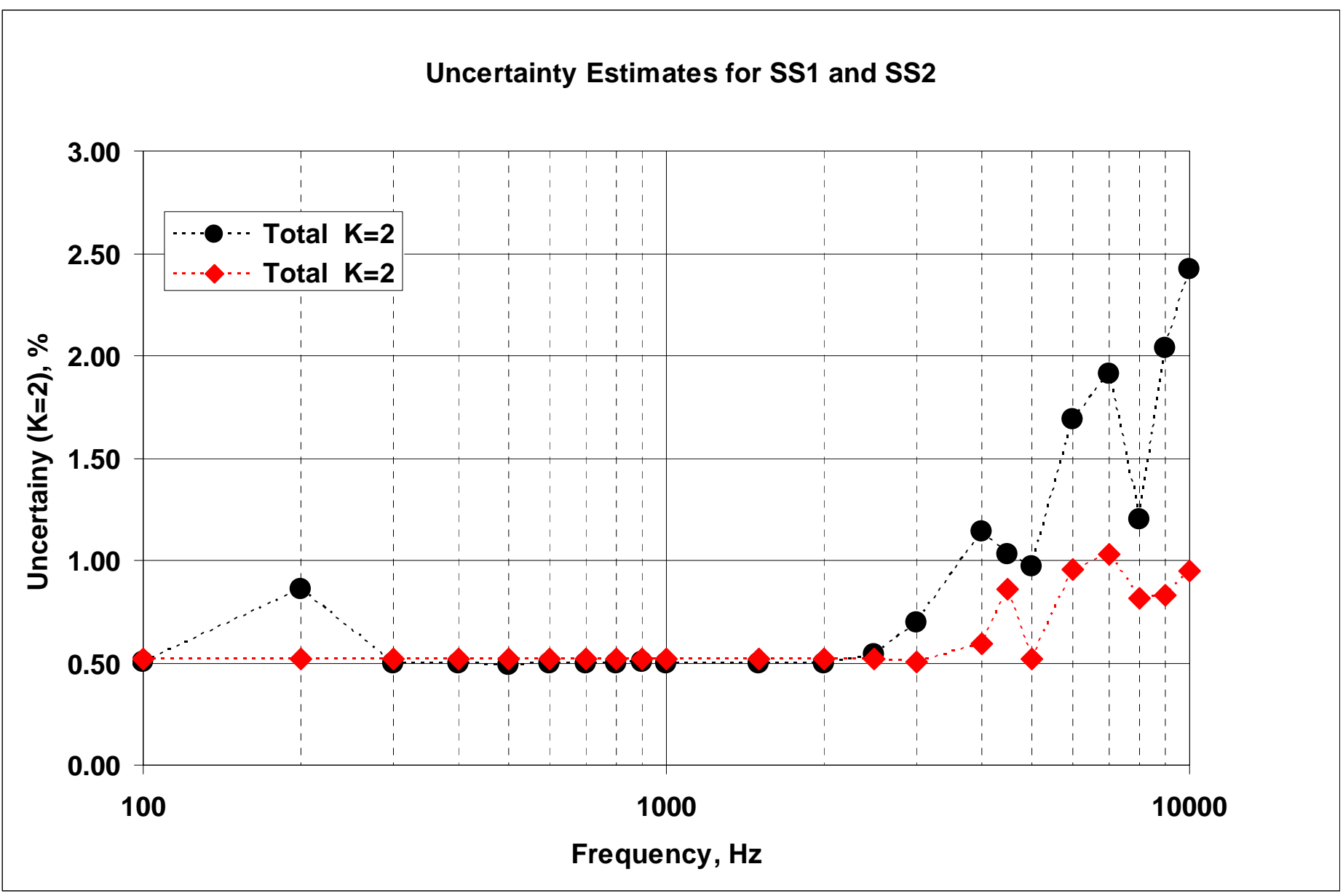


Comparison of Calibration Results for Same Accelerometer Tested on Both Super Shakers

$\begin{array}{rrrrrrrr}\text { Freq. (Hz) } & \text { RUN1 } & \text { RUN2 } & \text { RUN3 } & \text { RUN4 } & \text { RUN5 } & \text { SEPT 09 } & \text { SEPT 09 } \\ 100 & 0.009976 & 0.009978 & 0.009926 & 0.009965 & 0.01001 & 0.00997 & 0.01001 \\ 200 & 0.009976 & 0.009987 & & 0.010002 & 0.010001 & 0.00999 & 0.01004 \\ 300 & 0.009988 & 0.009983 & & 0.009995 & 0.009983 & 0.00999 & 0.01006 \\ 400 & 0.009988 & 0.009979 & 0.009982 & 0.009981 & 0.00998 & 0.00998 & 0.01003 \\ 500 & 0.009973 & 0.009979 & 0.009985 & 0.00998 & 0.009998 & 0.00998 & 0.01002 \\ 600 & & 0.009971 & 0.009981 & 0.00997 & 0.009969 & 0.00997 & 0.01005 \\ 700 & & 0.009979 & 0.009968 & 0.009981 & 0.009981 & 0.00998 & 0.01001 \\ 800 & 0.00998 & 0.009973 & 0.009981 & 0.009977 & 0.009969 & 0.00998 & 0.01001 \\ 900 & 0.010008 & 0.010002 & 0.010024 & 0.009995 & 0.00999 & 0.01000 & 0.01007 \\ 1000 & 0.010000 & 0.009993 & 0.00999 & 0.00998 & 0.009998 & 0.00999 & 0.01004 \\ 1500 & & 0.010017 & 0.010009 & 0.010017 & 0.010005 & 0.01001 & 0.01004 \\ 2000 & 0.010018 & 0.010022 & 0.010028 & 0.010025 & 0.010019 & 0.01002 & 0.01003 \\ 3000 & 0.010037 & 0.010036 & 0.010038 & 0.010023 & 0.010039 & 0.01003 & 0.01004 \\ 4000 & 0.010011 & 0.010052 & 0.010041 & 0.010104 & 0.01004 & 0.01005 & 0.01005 \\ 5000 & 0.010093 & 0.010069 & 0.010024 & 0.01009 & 0.010032 & 0.01006 & 0.01003 \\ 6000 & 0.010032 & 0.01001 & 0.010043 & 0.010028 & 0.009953 & 0.01001 & 0.01004 \\ 7000 & 0.010024 & 0.010019 & 0.01002 & 0.01009 & 0.010091 & 0.01005 & 0.01008 \\ 8000 & 0.010005 & 0.010046 & 0.010034 & 0.010021 & 0.010014 & 0.01002 & 0.01001 \\ 9000 & 0.009994 & 0.009988 & 0.009987 & 0.009998 & 0.009957 & 0.00998 & 0.01001 \\ 10000 & 0.009966 & 0.010045 & 0.009987 & 0.009984 & 0.010008 & 0.01000 & 0.01000\end{array}$


Plot of Comparison of Calibration Results for Same Accelerometer Tested on Both Super Shakers

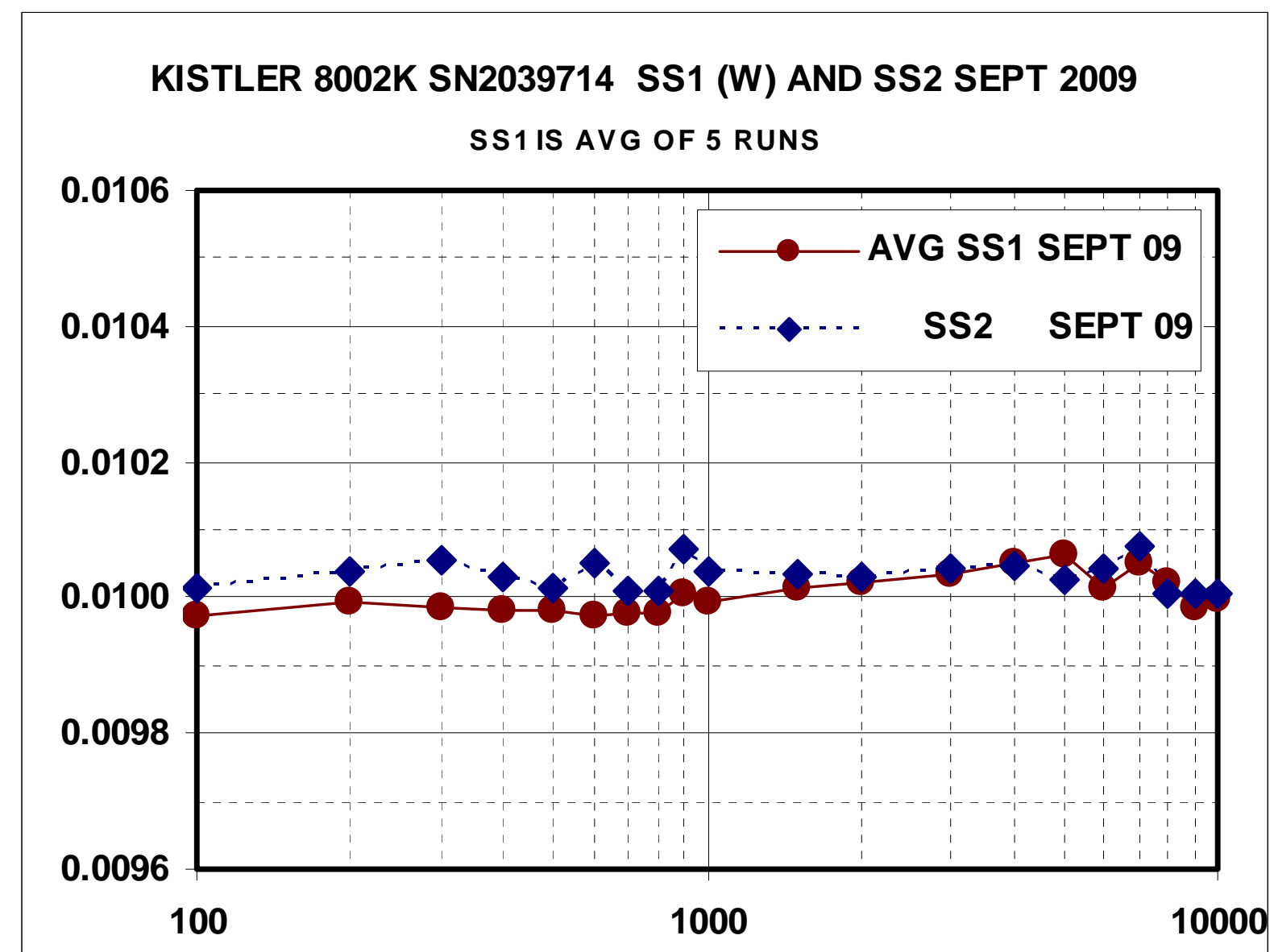

